1 An endothelial cell line infected by Kaposi's sarcoma associated herpes virus

2 (KSHV) allows the investigation of Kaposi's sarcoma and the validation of novel viral inhibitors in vitro and in vivo

4

5 Tatyana Dubich ${ }^{1}$, Anna Lieske$^{1}$, Susann Santag ${ }^{2,8}$, Guillaume Beauclair $^{2,8}$, Jessica Rückert ${ }^{2,8}$, Jennifer 6 Herrmann $^{3,8}$, Jan Gorges ${ }^{4}$, Guntram Büsche ${ }^{5}$, Uli Kazmaier ${ }^{4}$, Hansjörg Hauser ${ }^{1}$, Marc Stadler ${ }^{7,8}$, Thomas

7 F. Schulz ${ }^{2,8}$, Dagmar Wirth, ${ }^{1,6}$

${ }^{1}$ Model Systems for Infection and Immunity, Helmholtz Centre for Infection Research, Braunschweig/Germany

2 Institute of Virology, Hannover Medical School, Germany

3 Microbial Natural Products, Helmholtz Institute for Pharmaceutical Research, Saarbrücken/Germany

4 Institute of Organic Chemistry, Saarland University, Saarbrücken/Germany

${ }^{5}$ Institute of Pathology, Hannover Medical School, Germany

${ }^{6}$ Institute of Experimental Hematology, Hannover Medical School, Germany

7 Microbial Drugs, Helmholtz Centre for Infection Research, Braunschweig/Germany

8 German Centre for Infection Research, Hannover-Braunschweig/Germany

\title{
18 Correspondence
}

19 Prof. Dr. Dagmar Wirth

20 Model Systems for Infection and Immunity

21 Helmholtz Center for Infection Research

22 Inhoffenstr. 7

$23 \quad 38124$ Braunschweig 
26 Key words

27 KSHV, Drug validation, 3D culture system, Humanized mouse model, Novel antiviral drugs;

\section{Financial support}

29 The work was supported by the Deutsche Forschungsgemeinschaft (DFG, German Research Foundation)

30 via the Cluster of Excellence REBIRTH (From Regenerative Biology to Reconstructive Therapy) and the 31 SFB900 (Chronic Infection).

32 Conflict of interest

33 The authors declare that the research was conducted in the absence of any commercial or financial 34 relationships that could be construed as potential conflict of interest. Dagmar Wirth and Hansjörg Hauser (together with Tobias May) have filed a patent concerning the 36 technology for establishment of conditionally immortalized cell lines (PCT / EP2009 / 004854). 


\section{Abstract}

38 Kaposi's sarcoma-associated herpesvirus (KSHV) is the etiological agent of Kaposi's sarcoma (KS), a 39 tumor of endothelial origin predominantly affecting immunosuppressed individuals. Up to date, vaccines 40 and targeted therapies are not available. Screening and identification of antiviral compounds are 41 compromised by the lack of scalable cell culture systems reflecting properties of virus transformed cells 42 in patients. Further, the strict specificity of the virus for humans limits the development of in vivo models.

43 In this study we exploited a conditionally immortalized human endothelial cell line for establishment of in 44 vitro 2D and 3D KSHV latency models and the generation of KS-like xenograft tumors in mice. 45 Importantly, the invasive properties and tumor formation could be completely reverted by purging KSHV 46 from the cells, confirming that tumor formation is dependent on the continued presence of KSHV, rather than being a consequence of irreversible transformation of the infected cells. Upon testing a library of 260 natural metabolites we selected the compounds that induced viral loss or reduced the invasiveness of infected cells in 2D and 3D endothelial cell culture systems. The efficacy of selected compounds against KSHV induced tumor formation was verified in the xenograft model. Together, this study shows that the combined use of antiviral and antitumor assays based on the same cell line is predictive for tumor reduction in vivo and therefore allows faithful selection of novel drug candidates against Kaposi's sarcoma. 


\section{Introduction}

Up to $15 \%$ of human cancers are induced by oncogenic viruses [1]. One of the common oncogenic viruses is human herpesvirus 8 (HHV8), also known as Kaposi's sarcoma associated herpesvirus (KSHV). KSHV causes to at least three human malignances, namely Kaposi's sarcoma (KS), primary effusion lymphoma and the plasma cell variant of multicentric Castleman's disease. Kaposi's sarcoma is a tumor of endothelial origin, often manifesting itself in the skin, but also affecting liver, lungs, gastrointestinal tract and lymph nodes. While the prevalence of KSHV in the population varies geographically, with less than $10 \%$ in Northern Europe and $>40 \%$ in sub-Saharan Africa (reviewed in [2]), the tumor mainly occurs in immunocompromised or elderly patients. It is the most common neoplasm in untreated AIDS patients and in men in sub-Saharan Africa, as well as one of the most common malignancies in patients after organ transplantation [3]. Standard treatment options of Kaposi's sarcoma include surgery, chemotherapy and irradiation therapy. The response of HIV-positive patients with Kaposi's sarcoma to these therapies ranges from $22-80 \%$. Nevertheless, complete remission is rarely achieved [4].

Rapamycin, a natural product from actinobacteria, has emerged as a therapeutic compound of benefit for transplantation-associated KS and was shown to have antiangiogenic effects in a murine tumor model [5]. Several studies also suggest a selective sensitivity of KSHV-infected cells to rapamycin, as its molecular target mTOR is crucial for the survival of KSHV-infected cells and viral pathogenesis [6, 7]. This is in line with the observation that after renal transplantation in KSHV-infected patients the replacement of standard immunosuppressive drugs with rapamycin resulted in a reduction of Kaposi's sarcoma lesions [8]. However, rapamycin cannot be used as a standard therapy for Kaposi's sarcoma, since it is an immunosuppressive agent and suppression of the immune system is a key factor of Kaposi's sarcoma's development. This highlights the need for novel antiviral and antitumor agents.

KSHV-infected endothelial cells acquire spindle-like morphology [9] and undergo transcriptional reprogramming, referred to as endothelial-to-mesenchymal transition (EndMT) [10]. Most of the KSHV 
79 positive cells are latently infected and only a small proportion of the infected cells undergoes lytic reactivation $[11,12]$. Recently, several treatment options were suggested that target lytic reactivation of the virus (reviewed in [13]), whereas targeting the predominant latent stage is still a challenge. The investigation of the viral pathogenesis and identification of potential antiviral compounds are compromised by the lack of scalable and robust in vitro models that reflect the virus-induced changes observed in vivo. Moreover, due to the restricted host tropism of KSHV, small animal models have not been available so far. Murine gamma-2 herpesvirus 68 (MHV-68) was proposed as a model to mimic KSHV infection in the mouse [14]. Similarly to KSHV it establishes latent infection in B cells, which may lead to lymphoproliferative pathology $[15,16]$. However, MHV-68 fails to establish tumors of endothelial origin and therefore cannot be used as a small animal model for Kaposi's sarcoma.

We recently described conditionally immortalized human endothelial cells (HuARLT) that are permissive for KSHV infection $[17,18]$. The cell line preserves the important properties of primary endothelial cells in vitro and gives rise to functional blood vessels when transplanted into mice. After infection with KSHV the cells become spindle-like, lose endothelial properties and undergo transcriptional changes corresponding to EndMT, thereby reflecting the features as described for patients. Upon transplantation into immunocompromised mice the infected cells form lesions, histologically mimicking the lesions seen in Kaposi's sarcoma patients [18]. These properties suggest that this culture system may be suitable not only for the investigation of KSHV infection mechanisms but also for the validation of novel compounds. Similar to other herpesviruses, the KSHV genome adopts an episomal, circular state in infected cells with a range of viral loads. Recently, it has been reported that the viral load in skin lesions correlates with disease severity [19]. The results of the aforementioned study suggested that drug mediated reduction of the viral copy number might allow to reduce tumorigenicity of cells. In the current study, we established 2D and advanced 3D cell culture assays based on KSHV-infected endothelial HuARLT cells to screen a subset of 26 compounds from a natural compounds library for reducing the episomal viral copy number and the tumorigenic properties (sprouting activity) of KSHV- 
infected cells in in vitro cell culture systems. Selected compounds were subsequently tested for the ability to reduce the tumor size in xenotransplanted mice. Our analysis shows that the 3D sprouting activity correlates with tumor size observed in vivo. Importantly, we could prove that tumor formation in vivo depends on KSHV infection and is reverted when the virus is purged from these cells. Together, this endothelial cell system allows not only the identification of novel compounds that reduce viral load and/or tumorigenicity in vitro but also the validation of compounds against KS-like lesions in xenograft mice.

\section{$111 \quad$ Materials and Methods}

\section{Cell culture}

113 Human conditionally immortalized endothelial cells HuARLTs were generated in the lab of D. Wirth by 114 lentiviral transfer of a doxycycline controlled SV40 large T antigen and hTert expression cassette as 115 described before [17]. In 2D culture conditions, these cells proliferate in presence of $2 \mu \mathrm{g} / \mathrm{ml}$ doxycycline 116 while they stop proliferation in absence of doxycycline. The cells form 3D spheroids that can be 117 maintained in presence of doxycycline without increase of cell number [17]. rKSHV-HuARLT cells [18] 118 were derived upon infection of HuARLTs with recombinant KSHV.219 carrying the constitutively 119 expressed GFP and puromycin selection genes as well as RFP under control of a viral lytic promoter [20]. 120 HuARLT and rKSHV-HuARLT cells were cultivated on plates coated with $0.5 \%$ gelatin (G1393-100ML, 121 Sigma) in endothelial growth medium (EGM, CC-3124, Lonza) in a humidified normoxic atmosphere with $5 \% \mathrm{CO}_{2}$ in the presence of $2 \mu \mathrm{g} / \mathrm{ml}$ doxycycline. Maintenance cultures of rKSHV-HuARLT cells additionally contained $5 \mu \mathrm{g} / \mathrm{ml}$ puromycin, while all the experiments were performed in the absence of the selection drug. 
To assess cell viability upon treatment with standard drugs, rKSHV-HuARLT cells and HuARLT cells were seeded in 96-well plates (5000 cells/well) and incubated with $1.25 \mathrm{mg} / \mathrm{ml}$ rapamycin, $1.25 \mathrm{mg} / \mathrm{ml}$ FK506, $10 \mu \mathrm{M}$ BAY11-7085, or $12.5 \mu \mathrm{M}$ LY294002 for $72 \mathrm{~h}$ in absence of doxycycline. The maximal concentration of solvent did not exceed $0.5 \%$ of the total volume. Cell viability was measured using the WST-based Cell Counting Kit 8 (Sigma 96992-500TESTS-F) according to the manufacturer's instructions. In brief, cells were incubated with $100 \mu$ of fresh media and $10 \mu 1$ of the WST-8 solution at $37^{\circ} \mathrm{C}$ for 2-4 hours followed by absorbance measurement at $450 \mathrm{~nm}$ using TriStar ${ }^{2}$ LB 942 Modular Multimode Microplate Reader (Berthold Technologies). To calculate cell viability, the background values were subtracted and the data was normalized to DMSO-treated control values.

To determine the appropriate concentration for the new drugs, at least 4 concentrations per compound with 3 replicates were tested for cell viability. The drug concentrations that resulted in at least $80 \%$ viability are summarized in Supplementary Table 1 and were used for further experiments. $4000 \mathrm{rKSHV}-\mathrm{HuARLT}$ cells per well were seeded on a $0.5 \%$ agarose-coated 96 -well plate and cultivated at $37^{\circ} \mathrm{C}$ in a humidified normoxic atmosphere with $5 \% \mathrm{CO}_{2}$ in presence of doxycycline. After 24- or 48 hours, the formed aggregates (spheroids) were harvested and embedded either in a fibrin gel (which supports spheroid sprouting) or in a Matrigel matrix (which provides robust matrix stability for extended cultivation times as required for copy number analysis). 2D cultures: rKSHV-HuARLT cells were seeded on a 12 -well plate $\left(1 \times 10^{5}\right.$ cell/well $)$ and treated with the test compounds in absence of doxycycline and puromycin for 14 days. 3D cultures: for every well 6 to 8 spheroids were mixed with $0.7 \mathrm{mg} / \mathrm{ml}$ fibrin $(341576$, Calbiochem), 0.4\% methylcellulose (M0512, Sigma) and $0.5 \mathrm{U} / \mathrm{ml}$ thrombin (605190-100U, Merck Millipore) in EGM 
medium supplemented with $2 \mu \mathrm{g} / \mathrm{ml}$ doxycycline. Matrigel (Growth factor reduced, 354230 Becton Dickinson) was added to the spheroid mixture in a ratio of $1: 1.100 \mu 1$ of the gels were cast onto a 96-well plate and allowed to solidify for $30 \mathrm{~min}$ at $37^{\circ} \mathrm{C} .100 \mu \mathrm{l}$ of EGM supplemented with selected compounds was added on top of the gels (concentration of compounds is given in Supplementary Table 1). Media was changed and fresh compounds added to the media every 3-4 days in both 2D and 3D culture. After 14 days of treatment, DNA was isolated from cells cultured under 2D or 3D culture according to a protocol described before [21]. Briefly, the cells were lysed using modified Bradley's buffer with Proteinase K (19133, Qiagen) at $55^{\circ} \mathrm{C}$ overnight. Cellular DNA was precipitated in $75 \mathrm{mM}$ sodium acetate in $96 \%$ ethanol solution. The pellet was washed with $70 \%$ ethanol, dried and suspended in nuclease-free water. qPCR was performed at $58^{\circ} \mathrm{C}$ annealing temperature using SsoFast ${ }^{\mathrm{TM}}$ EvaGreen ${ }^{\circledR}$ Supermix (1725204, Biorad) in a LightCycler 480 II (29376, Roche). The data were analyzed using Light Cycler 480 software 1.5. Viral DNA was detected using LANA specific primers and normalized to cellular DNA detected by the ACTB specific primer pair (Supplementary Table 2). The number of viral copies per cell was extrapolated using a modified $\Delta \mathrm{Cp}$ method, taking into account that the cellular genome harbors 2 copies of the ACTB gene:

Viral copy number $/$ cell $=2 * 2^{C p_{L A N A}-C p_{A C T B}}$ Bio). The mixtures were cast onto a 96 -well plate and allowed to solidify for 30 minutes at $37^{\circ} \mathrm{C}$. The respective compounds were added in EGM media using the concentrations listed in Supplementary Table 1 and incubated for 5 days. Quantification of spheroid sprouting was performed from fluorescence 
representative focus plane was measured and normalized against the spheroid's core area (sprouting index).

Viral gene expression (RT-qPCR)

Total RNA was isolated from $5 \times 10^{5}$ rKSHV-HuARLT cells using RNAeasy mini kit (74106, Quiagen) according to the manufacturer's instructions and measured with the ND-1000 spectrophotometer (Nanodrop Technologies). cDNA was synthetized from $500 \mathrm{ng}$ of RNA using Reverse-Aid First Strand cDNA Synthesis Kit (K1622, Fisher Scientific) according to the manufacturer's instructions. Quantitative PCR was performed as described above using the primers specified in Supplementary Table 3. Relative expression of viral genes in relation to cellular ACTB was calculated using the standard $\Delta \mathrm{Cp}$ method.

Natural compound library and screening of natural compound library against KSHV lytic replication

The natural compounds used in this study have been compiled at the Helmholtz Center for Infection Research and the Helmholtz Institute for Pharmaceutical Research Saarland in a ready-to-screen library that is available for screenings to evaluate their utility in various biological assays. The chemical synthesis of pretubulysin D was described before [23]. For assays on endothelial cells we specifically selected derivatives from active compound classes that are feasible for in vivo studies due to availability and overall better characterization in terms of biological effects in other in vitro and in vivo experiments. Brk.219, a BJAB cell line stably infected with rKSHV.219 [24, 25] was used to screen a library of 260 natural compounds. Brk.219 cells were seeded into round bottom 96 well plates at a density of $10^{5}$ cells per well in $100 \mu \mathrm{RPMI}$ medium. Compounds were added at a final concentration of $10 \mu \mathrm{M}$ and the viral lytic cycle induced by the addition of an antibody to human $\operatorname{IgM}$ on the BJAB cell surface as described [24] Forty-eight hours later the supernatant of individual wells was collected and used to infect HEK293 cells. After a further 48 hours the number of GFP positive HEK293 cells was quantified by a Biotek fluorescence reader. The viability of the treated Brk.219 cells was determined by MTT assay. 
All compound tests on rKSHV-HuARLT cells were performed in non-proliferating cells. In particular, 2D culture based tests on rKSHV-HuARLT cells were performed in absence of doxycycline and in absence of puromycin. As shown before, long term cultures of rKSHV-HuARLT cells in 3D requires the presence of doxycycline, which does not lead to proliferation but rather is required for maintenance of spheroids over time [18]. Accordingly, compound test in 3D cultures were performed in presence of doxycycline.

\section{Flow cytometry}

$5 \times 10^{5}$ rKSHV-HuARLT or HuARLT cells were washed and detached with trypsin/EDTA and resuspended in PBS supplemented with 2\% FCS. Flow cytometric analysis was performed on a FACSCalibur $^{\mathrm{TM}}$ analyzer (Becton-Dickinson). Non-infected cells were used as a negative control. The data were processed with FlowJo v10 software. Immunofluorescence microscopy rKSHV-HuARLT cells were plated on $0.5 \%$ gelatin-coated cover glass slips and fixed for 20 min with $4 \%$ formaldehyde in PBS followed by permeabilization with $0.5 \%$ Triton X-100 in PBS for $10 \mathrm{~min}$. Blocking of the samples was done in PBS supplemented with 2\% BSA for $1 \mathrm{~h}$. The coverslips were stained with mouse monoclonal anti-LANA (NCL-HHV8-LNA, Leica) antibodies in PBS with $0.1 \%$ saponin Quillaja sp. (S4521, Sigma) at $4{ }^{\circ} \mathrm{C}$ overnight. Staining with Cy3-labeled secondary goat antimouse antibodies (Dianova) was performed in in PBS with $0.1 \%$ saponin at RT for $1 \mathrm{~h}$. Coverslips were mounted on glass slides with Fluoroshield ${ }^{\mathrm{TM}}$ containing DAPI (F6057-20ML, Sigma) and incubated at room temperature overnight. Images were acquired using a Zeiss LSM META confocal laser scanning microscope. Brightness and contrast were adjusted using ImageJ software.

\section{Mouse experiments}

rKSHV-HuARLT cells were transplanted to mice as described before [18]. In brief, $1.2 \times 10^{6}$ cells were seeded into each well of AggreWell ${ }^{\mathrm{TM}} 400$ (27945, Stemcell Technologies), centrifuged for 3 minutes at 
$100 \mathrm{~g}$ and cultivated for 3 days at $37^{\circ}$ C. 400 spheroids were used for each matrigel implant containing $0.2 \%$ methyl cellulose, $3 \mathrm{mg} / \mathrm{ml}$ fibrinogen in EGM media supplemented with $10 \mu \mathrm{g} / \mathrm{ml} \mathrm{FGF} \mathrm{(100-18B-}$ 250, PeproTech), $0.5 \mu \mathrm{g} / \mathrm{ml}$ VEGF (RCPG246, Randox), $1 \mathrm{U} / 1$ thrombin (605190-100U, Merck 224 Millipore) and $300 \mu 1$ of Matrigel HC (high protein, growth factor reduced, 354263, Becton Dickinson). The mixture was injected subcutaneously to $\operatorname{Rag} 2^{-/-} \mathrm{\gamma c}^{-/-}$mice. $1 \mathrm{mg} / \mathrm{ml}$ of doxycycline was added to the drinking water for the whole experiment.

Starting from day 1 after implantation, the mice were treated with the drugs according to the route, dose and regime adapted from previous studies (Supplementary table 3 for details).

229 Animal experiments were performed in accordance with the ethical laws and were approved by the local 230 authorities (permission number 33.19-42502-04-17/2480).

\section{Immunohistological stainings}

After 4 weeks of treatment, Matrigel implants were extracted and fixed with $4 \%$ formalin, embedded in paraffine, sectioned and stained with hematoxylin-eosin. Diameters of the lesions were measured on histological sections, stained for human vimentin. Human endothelial cells were marked applying in situ hybridization technique (ALU) and immunohistochemistry. The ALU probe was purchased from Ventana/Roche Diagnostics GmbH applying Ventana ISH detection kit. The following antibodies and dilutions were applied in order to mark the atypical endothelial cells using the BenchMark Ultra staining machine (purchased from Roche): antibody against CD141 (Serotec, 1:40), CD34 (Immunotech, 1:50), GFP (Santa Cruz Biotechnology inc; 1:10), HHV8 (LANA) (Novocastra / Menarini; 1:30); vimentin (Dako; 1:100) 


\section{Results}

\section{KSHV establishes latency in conditionally immortalized human endothelial}

cells

In order to develop assays for the identification of novel therapeutic agents against KSHV, we employed HuARLT cells, which maintain the properties of endothelial cells and which are characterized by doxycycline dependent growth in 2D culture conditions [18]. Infection with rKSHV.219 encoding puromycin acetyl transferase and GFP [20] allowed for the selection and monitoring of infected cells. If cultivated under selection pressure, the rKSHV.219-infected HuARLT cells (rKSHV-HuARLT) display the characteristic spindle-like morphology and show punctuated localization of the latency associated nuclear antigen LANA in the nuclei, indicating the association of viral episomes with the cellular chromosomes (Fig. 1a). To investigate viral maintenance in the absence of selection, rKSHV-HuARLT cells were cultured in 2D culture conditions in absence of doxycycline and puromycin, followed by flow cytometry analysis of GFP expression. Under these conditions, GFP expression only slowly declined over time, with nearly $80 \%$ of cells still being positive for GFP after 16 days (Fig. 1b). At the same time, viral DNA decreased from $9.19 \pm 1,88$ to $4,61 \pm 0,61$ copies per cell as determined by qPCR (Fig. 1c), indicating loss of viral copies during cultivation.

In order to determine the predominant state of the viral life cycle in infected rKSHV-HuARLT cells, we evaluated the expression level of prototypic latent and lytic genes relative to cellular ACTB using RTqPCR. The infected cells showed elevated levels of latent mRNAs, such as LANA, vCyclin, vFLIP and kaposin, whereas mRNAs of viral lytic genes, such as PAN, K-bZIP, ORF50, ORF45 were expressed at lower levels or not at all, indicating that the infected cells had established latency (Fig. 1d)

The crucial role of various cellular signaling pathways was previously highlighted for survival of virusinfected cells [26]. To confirm the relevance of these pathways in the established cell system, we 
investigated the viability of infected cells upon inhibition of the respective pathways. We observed differential cell viability upon treating infected and non-infected cells with rapamycin, BAY11-7085 and LY294002, i.e. agents that block mTOR, NF-kB and PI3K pathways, respectively (Fig. 1e). FK506, which is structurally related to rapamycin and shares a number of molecular targets with it, except for mTOR, was used as a negative control in this assay. In agreement with previous studies in primary cells [26] these data suggest that mTOR, PI3K and NF-kB pathway are important for survival of KSHVinfected HuARLT cells.

In conclusion, these results indicate that KSHV-infected HuARLT cells reflect the status of virus-infected cells in vivo and represent a valid model to identify novel active compounds.

\section{Purging cells from virus abolishes the tumorigenic phenotype}

We asked if the loss of the viral genome could revert the phenotype of infected cells and abrogate tumor formation in vivo. To this end, we expanded rKSHV-HuARLT cells for 38 days in presence of doxycycline but without puromycin. Following the prolonged cultivation GFP negative cells were isolated by FACS (designated as KF-HuARLT cells, Fig. S1). Analysis of the viral copy number in KFHuARLT cells confirmed a significant reduction of viral genomes down to $0.07 \pm 0.04$ copies per cell indicating the absence of the KSHV genome in the vast majority of these cells (Fig. 1c).

To investigate the tumor potential of KF-HuARLT cells, KF-HuARLT as well as the control rKSHVHuARLT and HuARLT cells were aggregated to spheroids and transplanted into immunocompromised Rag2 $^{-/} \gamma \mathrm{c}^{-/-}$mice according to a previously described protocol [18] (Fig. 2a). After 4 weeks, the plugs were isolated. Sections were stained for human vimentin and the morphology of transplanted cells was examined via microscopy. The virus-infected rKSHV-HuARLT cells formed lesions that were positive for virus encoded GFP and LANA as well as for human ALU sequences (Fig. 2b). Further, the lesions were characterized by spindle cells as well as expression of human CD141 (thrombomodulin) which is found on Kaposi's sarcoma cells [27], together reflecting the early patch stage of human KS (Fig. 2b). In 
contrast, transplantation of KF-HuARLT cells resulted in engraftment of individual cells, however, no KS-like lesion could be found (Fig. 2c). Thus, elimination of viral genomes from KSHV-infected HuARLT cells completely reverted the tumorigenic phenotype and impaired the formation of lesions indicating that continuous expression of KSHV genes is crucial for tumor formation. Further, this raises the hypothesis that the reduction of viral copy number might be a useful parameter to identify antitumor compounds.

\section{Establishment of in vitro assays for compound validation}

We evaluated the drug-induced reduction in viral load upon culturing the rKSHV-HuARLT cells in 2D culture conditions for 14 days in the absence of puromycin. To validate the loss of the episomal viral copies as a readout for antiviral drug selection, we used glycyrrhizic acid (GA). GA downregulates LANA, the protein responsible for tethering viral episomes to the cellular genome, and thereby reduces the viral load of infected cells [28]. rKSHV-HuARLT cells were treated with GA for two weeks. Then, GFP expression was determined and the viral copy number per cell was assessed by qPCR. As depicted in Figure 3a and Fig. S2, the GA treatment induced substantial viral loss as determined by both readouts. In contrast, treatment of the infected cells with phosphonoformic acid (PhA), an inhibitor of lytically expressed viral DNA polymerase, did not have an effect on the viral copy number and GFP expression. These data support the observation that the cells are predominantly latently infected and lytic reactivation does not play a role in viral maintenance in $2 \mathrm{D}$ culture.

Several studies indicate that $3 \mathrm{D}$ cell culture is beneficial for viral gene expression in lymphatic endothelial as well as in B cells and KSHV-induced cell transformation is more pronounced under these conditions $[10,29]$. Therefore, we tested if viral maintenance is better reflected by $3 \mathrm{D}$ culture conditions. We observed that after cultivation of cells for 2 weeks under 3D conditions in the absence of selection, the viral load was slightly but significantly higher when compared to standard 2D conditions, with about $10.9 \pm 1.12$ virus copies per cell in $3 \mathrm{D}$ versus $5.5 \pm 0.67$ in $2 \mathrm{D}$ conditions $(\mathrm{p}=0.0009)$. Interestingly, treatment of 3D spheroid cultures with GA but also with $\mathrm{PhA}$ resulted in a significant reduction of the 
viral load (Fig. 3b). The fact that PhA has an impact on viral copy number suggests that maintenance of virus in 3D conditions involves lytic phases of virus infection and is partially due to viral proteins expressed during the lytic replication cycle.

For evaluation of the invasiveness of KSHV-infected cells upon compound treatment we adapted a 3D culture system previously described for KSHV-infected primary endothelial cells [10]. rKSHV-HuARLT cells were first aggregated to spheroids and then embedded in a fibrin matrix. Three days after embedding, the KSHV-infected cells exhibited pronounced sprouting and invaded into the surrounding matrix (Fig. 3d). To validate the specificity of the assay with respect to KSHV, we evaluated the effect of $\mathrm{GA}, \mathrm{PhA}$ and rapamycin on sprouting in $3 \mathrm{D}$ culture conditions. To that end, the embedded spheroids were treated with the compounds for five days. The effect of the compounds was evaluated by microscopy and quantified by measuring the area covered by sprouts and relating it to the spheroid body area in the same section (sprouting index, Fig. S3). Quantification revealed a significant reduction of sprouting for GA and Rapamycin, which impair viral maintenance and provide antiangiogenic activity, respectively [5]. In contrast, treatment with the control drugs FK506 and PhA did not reduce the sprouting activity (Fig. 3c). Together, the three assay systems are appropriate to test compounds for antiviral and/or antitumor activities.

\section{Evaluation of novel compounds for viral loss in 2D and 3D culture models}

To search for novel anti-KSHV agents that would reduce viral copy number and/or the sprouting of KSHV-infected HuARLT cells, we studied a library of 260 natural compounds. This library mainly consists of secondary metabolites from Myxobacteria, that were selected by previous natural product screening programs [30]. We used a previously described reactivation assay in KSHV-infected BJAB cells (Brk.219 cells) $[24,25]$ to narrow down the list of candidates to be tested in our 3D sprouting assay. We treated Brk.219 cells with the natural compounds in this library and used supernatants from compound-treated reactivated Brk.219 cells to infect HEK 239T cells. Infection rate of HEK 293 was measured via fluorescence microscopy. Fig. 4a indicates the compounds that showed reduction of GFP 
expression in HEK293 cells and therefore inhibited KSHV lytic replication in Brk.219 cells, while at the same time exhibiting low to moderate toxicity (Fig. S4) in Brk.219 cells.

On the assumption that compounds capable of inhibiting lytic KSHV reactivation in Brk.219 cells might do so either because they interfere with the lytic cycle or reduce the number of latent KSHV genomes in this cell line, and since the KSHV lytic cycle is known to contribute to tumorigenesis (reviewed in [31]), we investigated if compounds with activity in the Brk.219 assay would also reduce the viral load in latently KSHV-infected endothelial HuARLT cells. We selected 26 compounds including pretubulysin D, a chemically accessible precursor of tubulysins [23]. First, we tested their impact on viral copy number in KSHV-infected HuARLT cells using drug concentrations that had little influence on cell viability $(>80 \%$ or more viable cells, Fig. S5 and Supplementary Table 1). The KSHV latently infected HuARLT cells were cultured in 2D conditions in absence of doxycycline and puromycin and treated with the compounds for 14 days followed by evaluation of viral copy number by qPCR. Out of the 26 compounds tested, seven showed a reduction of the cellular viral load to $75 \%$ : epothilon E, myxochelin A, eliamid, saframycin Mx1, stigmatellin F, chondramides B and C (Fig. 4b). This set of compounds was tested under the more physiologically relevant 3D conditions. To this end, matrigel embedded spheroids formed from KSHV-infected cells were treated for 14 days with the compounds and then evaluated for the viral copy number per cell. A total of nine compounds were identified that showed a reduction to $60 \%$. In addition to five compounds that already showed positive effects in the 2D assay (epothilon E, myxochelin A, eliamid, chondramides B and C) four compounds showed copy number reductions in 3D culture only: trichangion, pellasoren, tubulysin A, tubulysin X (Fig. 4c).

To investigate whether the selected compounds have an impact on the cell invasiveness in $3 \mathrm{D}$ culture conditions, we evaluated their effect on the cell's sprouting activity. To this end fibrin gel embedded spheroids produced from rKSHV-HuARLT cells were treated with the compounds for 5 days followed by evaluation of the sprouting index. Overall, 13 compounds significantly reduced sprouting to $60 \%$ or less 
in the 3D sprouting assay, indicating pronounced reduction in the invasive potential of the cells (Fig. 4d).

In Fig. 4e, the results of the three in vitro assays are summarized.

\section{Validation of hits in vivo}

We selected four compounds with various degrees of inhibition in the three in vitro test systems, for which pharmacokinetic as well as biavailability data were available, and investigated their ability to reduce the formation of KS-like lesions in the xenograft model. Chondramid B was chosen as a compound that significantly reduced the viral load in $2 \mathrm{D}$ and $3 \mathrm{D}$ culture, but with a moderate effect on the sprouting index. In contrast, pretubulysin D and epothilon B were selected for their strong effect on sprouting but no significant effect on the viral copy number. As a drug with minor activities we selected soraphen A. In addition, we used the mTOR inhibitor rapamycin (and its control FK506) as well as PhA with known antiviral activities. Starting directly after transplantation of the virus-infected cells, the mice were treated with the respective drugs for 4 weeks. Then, the mice were sacrificed and the plugs were isolated. The lesion size was assessed by microscopy analysis of vimentin stained sections.

While the control compound FK506 showed no significant effect on tumor size, a certain reduction of lesion size down to about $70-80 \%$ of the size of untreated controls was found in mice treated with rapamycin and PhA. Interestingly, a comparable reduction of tumor size was observed for chondramid B and rapamycin which was accompanied by a reduction in viral copy number (Fig. S6). However, the strongest impairment was observed for epothilon B and pretubulysin D which resulted in a complete regression of the tumor growth (Fig. 5a and 5b). Moreover, the drug-mediated reduction of invasiveness of rKSHV-HuARLT cells in the 3D cell culture conditions strongly correlates with tumor size reduction $\left(\mathrm{R}^{2}=0,9266\right.$, Fig. $\left.5 \mathrm{c}\right)$, highlighting the predictive power of the in vitro system with respect to tumor formation in vivo. 


\section{Discussion}

In this study, we exploited the properties of the previously developed conditionally immortalized human endothelial cell line HuARLT [18] upon infection with KSHV. This cell line reflected the consequences of infection on various levels. Similar to cells in Kaposi's sarcoma lesions $[11,12]$ the majority of rKSHV-HuARLT cells established latency upon infection with KSHV. Further, their growth critically depended on PI3K/Akt/mTOR and NF-kB pathways, properties that were shown to provide survival advantage to infected cells [26]. We previously reported that latently-infected HuARLT cells reduce expression of endothelial markers and upregulate mesenchymal markers [18] and thus reflect the endothelial-to-mesenchymal transition observed upon infection of primary human endothelial cells [10]. Finally, infected rKSHV-HuARLT cells gave rise to KS-like lesions upon transplantation into mice, opening the opportunity to investigate the fate of infected cells in vivo. Together, this makes the cell line suitable for investigation of features provoked by KSHV infection and for development of novel compounds in vitro and in vivo.

We hypothesized that reduction of viral load within KSHV-infected cells would result in the loss of their tumorigenic potential. This hypothesis is supported by the observation that the viral load within tumor cells correlates with the tumor burden in the patients and reflects the severity of the disease [19] while (at the same time) the plasma level of KSHV is a poor prognostic or diagnostic biomarker for KS $[19,32]$. To test this hypothesis, we established a virus-free cell population by passaging rKSHV-HuARLT cells in absence of selection pressure. We observed that indeed the virus-purged cell population lost their tumorigenic potential upon transplantation in vivo. While establishment of KS-like lesions has also been demonstrated in nude mice with the help of other cell systems [33-36], this study confirms that tumorigenicity of the rKSHV-HuARLT cell system is virus-dependent. Thus, viral proteins are essential for inducing the tumorigenic phenotype which is reverted when the cells are depleted from the virus, making this system of particular value for both the investigation of virus induced tumorigenicity and for 
412 the validation of antiviral compounds specifically acting against KSHV and KSHV induced tumor

413 formation.

414 In depth characterization indicated that the virus established a strictly latent stage in 2D culture and viral 415 maintenance was not affected through blocking the lytically expressed viral DNA polymerase by PhA. In contrast, maintenance of the virus in 3D cell culture at least partially depended on lytic reactivation, as indicated by reduction of viral copy number in presence of $\mathrm{PhA}$. This observation corresponds to recent studies indicating that $3 \mathrm{D}$, but not $2 \mathrm{D}$ cell culture conditions supports lytic reactivation of the virus [10, 29 ] and indicates that the $3 \mathrm{D}$ cell culture allows validation of the compounds targeting not only latent maintenance, but also lytic replication of the KSHV. 3D sprouting was shown to be useful to study ex vivo angiogenesis [22] as well as the evasion [37] and KSHV-induced invasion of lymph endothelial cells [10]. We showed that impairing viral replication by $\mathrm{PhA}$ had only a minor effect on $3 \mathrm{D}$ sprouting. In line with this, $\mathrm{PhA}$ could also slightly reduce the formation of KS lesions in vivo to a certain extent, although this was statistically not significant. This observation is in line with clinical observations showing that drugs targeting viral lytic replication prevent the formation of KS lesions. Still, inhibitors of lytic replication fail to reduce established lesions and thus may not be suitable for treatment of KS (reviewed in [13]). This might suggest that inhibition of viral replication alone is not sufficient to block invasiveness of the infected cells and illustrates the limitation of assay systems relying purely on viral copy reduction. Various viral proteins have been shown to contribute to KSHV-induced invasiveness, including factors expressed in the latent (e.g. LANA [38, 39], vFLIP [40], vCyc [41]) and the lytic (e.g. IL-6 [42], vGPCR [43-45], K1 [46], K15 [47, 48]) phase of the viral life cycle. Screening for drugs only according to their ability to reduce KSHV reactivation would thus miss potential KS inhibitors that target KSHV-induced invasiveness and angiogenesis and that would not necessarily affect viral lytic replication. This highlights the need for predictive in vitro screening systems that consider both, viral load as well as cellular invasiveness. Based on KSHV-HuARLT cells different phenotypic screening systems could be 
established, which allow investigation of the drugs targeting viral maintenance and affecting in sprouting and angiogenesis.

With the KSHV-HuARLT based assays we validated a set of natural compounds and challenged the 440 selected compounds with anti-viral or anti-sprouting activity in a humanized mouse model. As a result, three compounds were identified with comparable or even higher potency to reduce the size of KS lesions than rapamycin, a drug that is in clinical use for KSHV-infected organ transplant recipient with KS. Pretubulysin D and epothilon B were described before as antitumor and antimetastatic agents acting primarily on cellular cytoskeleton $[49,50]$. Both compounds drastically reduce sprouting and tumor size in vivo, but have no effect on viral copy number. This suggests that they mainly act via the inhibition of microtubule formation as described previously, rather than targeting pathways relevant for viral maintenance. In contrast, chondramid B significantly reduced both the viral load in vitro and the tumor size in vivo. The data suggest that the compound might interfere with viral maintenance. Previously, it was shown that chondramid B promotes actin polymerization, thereby also diminishing angiogenesis in vitro and in vivo [51]. It remains to be elucidated if the reduction of viral load by chondramid B depends on its function on actin or is a result of the interaction with other molecular targets. Also, the reduction in tumor size in the presence of chondramid B might not be exclusively attributed to its effect on the virus, but could be a combination of both, the reduction of viral load and deteriorating the nutrition of the tumor by impairing angiogenesis. Interestingly, while purging the virus from cells could in principle abolish the tumorigenic properties of infected cells, the strongest anti-tumor effect in vivo was observed for pretubulysin and epothilone which have a direct antiproliferative and antiangiogenic effect. This might suggest that reduction of viral load alone has a limited therapeutic potential but might be more efficient as a part of combined antitumor and antiviral therapies. However, more compounds as well as combination protocols would need to be evaluated to draw a general conclusion. Of note, in this study we investigated effects of anti-KSHV 
treatment applied together with the transplantation of the infected cells, which does not reflect a course of tumor development in patients. Evaluating the performance of the drugs on pre-existing KSHV induced tumors will allow the assessment of the drugs in clinical-like situations. The mouse model we introduce here would be of particular benefit for such advanced studies. Animal tests play a crucial role in drug development, because they allow not only the confirmation of selective drug activity, but also assess its pharmacokinetics, pharmacodynamics, toxicity and safety in vivo. Humanized mouse models expand the toolbox and allow us to study human-specific diseases, like Kaposi's sarcoma, as well. In this regard, advanced and predictive in vitro systems can improve drug development by providing meaningful tools for screening of large compound libraries in vitro and prevalidating the performance, thereby reducing the amount of required experimental animals and lowering 472 the costs of drug development. Our findings highlight the potential of the assay system to identify compounds that target pathways relevant for supporting viral latency in vitro and to evaluate the reduction of tumor growth in vivo established from the same cell line. Since the combined use of antiviral and antitumor assays is indicative for antitumor activity in vivo, it has the potential for faithful pre-selection of active compounds in vitro to reduce the number of experimental animals and ensure compliance with $3 R$ principles, i.e. the replacement of animal experiments, reduction amount of animals used, and refinement of experimental conditions. 


\section{Acknowledgments}

483 T.D. acknowledges support by the HZI Grad School. Further, we thank the central animal facility (TEE)

484 at HZI for excellent support.

485 


\section{References}

1. zur Hausen H (2001) Oncogenic DNA viruses. Oncogene 20:7820-7823 . doi: 10.1038/sj.onc.1204958

2. Mesri E a, Cesarman E, Boshoff C (2010) Kaposi's sarcoma and its associated herpesvirus. Nat Rev Cancer 10:707-19 . doi: $10.1038 / \mathrm{nrc} 2888$

3. Raeisi D, Madani SH, Zare ME (2013) Kaposi's Sarcoma after Kidney Transplantation: a 21-Years Experience. 7:

4. Union for International Cancer Control (2014) Review of Cancer Medicines on the WHO List of Essential Medicines: Kaposi's Sarcoma

5. Guba M, von Breitenbuch P, Steinbauer M, et al (2002) Rapamycin inhibits primary and metastatic tumor growth by antiangiogenesis: involvement of vascular endothelial growth factor. Nat Med 8:128-135 . doi: $10.1038 / \mathrm{nm} 0202-128$

6. Ganem HHC and D (2013) A unique herpesviral transcriptional program in KSHV-infected lymphatic endothelial cells leads to mTORC1 activation and rapamycin sensitivity. Cell Host Microbe 144:724-732 . doi: $10.1038 /$ jid.2014.371

7. Roy D, Sin SH, Lucas A, et al (2013) MTOR inhibitors block kaposi sarcoma growth by inhibiting essential autocrine growth factors and tumor angiogenesis. Cancer Res 73:2235-2246 . doi: 10.1158/00085472.CAN-12-1851

8. Stallone G, Schena A, Infante B, et al (2005) Sirolimus for Kaposi's sarcoma in renal-transplant recipients. N Engl J Med 352:1317-1323 . doi: 10.1056/NEJMoa042831

9. Alkharsah KR, Singh VV, Bosco R, et al (2011) Deletion of Kaposi's sarcoma-associated herpesvirus FLICE inhibitory protein, vFLIP, from the viral genome compromises the activation of STAT1-responsive cellular genes and spindle cell formation in endothelial cells. J Virol 85:10375-88 . doi: 10.1128/JVI.0022611

10. Cheng F, Pekkonen P, Laurinavicius S, et al (2011) KSHV-initiated notch activation leads to membranetype-1 matrix metalloproteinase-dependent lymphatic endothelial-to-mesenchymal transition. Cell Host Microbe 10:577-590 . doi: 10.1016/j.chom.2011.10.011 
512 11. Dittmer DP (2003) Transcription profile of Kaposi's sarcoma-associated herpesvirus in primary Kaposi's 513 sarcoma lesions as determined by real-time PCR arrays. Cancer Res 63:2010-2015

514 12. Hosseinipour MC, Sweet KM, Xiong J, et al (2014) Viral profiling identifies multiple subtypes of Kaposi’s sarcoma. MBio 5:e01633-14 . doi: 10.1128/mBio.01633-14

13. Coen N, Duraffour S, Snoeck R, Andrei G (2014) KSHV targeted therapy: An update on inhibitors of viral lytic replication. Viruses 6:4731-4759 . doi: 10.3390/v6114731

14. Virgin HW 4th, Latreille P, Wamsley P, et al (1997) Complete sequence and genomic analysis of murine gammaherpesvirus 68. J Virol 71:5894-5904

16. Dong S, Forrest JC, Liang X (2017) Murine Gammaherpesvirus 68: A Small Animal Model for Gammaherpesvirus-Associated Diseases. Adv Exp Med Biol 1018:225-236 . doi: 10.1007/978-981-10$5765-6$

19. Boivin G, Gaudreau A, Routy JP (2000) Evaluation of the human herpesvirus 8 DNA load in blood and

20. Vieira J, O'Hearn PM (2004) Use of the red fluorescent protein as a marker of Kaposi's sarcoma-associated

22. Shao Z, Friedlander M, Hurst CG, et al (2013) Choroid sprouting assay: an ex vivo model of microvascular angiogenesis. PLoS One 8:e69552 . doi: 10.1371/journal.pone.0069552

23. Ullrich A, Chai Y, Pistorius D, et al (2009) Pretubulysin, a potent and chemically accessible tubulysin precursor from Angiococcus disciformis. Angew Chem Int Ed Engl 48:4422-4425 . doi: 
10.1002/anie. 200900406

24. Kati S, Tsao EH, Gunther T, et al (2013) Activation of the B cell antigen receptor triggers reactivation of latent Kaposi's sarcoma-associated herpesvirus in B cells. J Virol 87:8004-8016 . doi: 10.1128/JVI.0050613

25. Kati S, Hage E, Mynarek M, et al (2015) Generation of high-titre virus stocks using BrK.219, a B-cell line infected stably with recombinant Kaposi’s sarcoma-associated herpesvirus. J Virol Methods 217:79-86 . doi: $10.1016 /$ j.jviromet.2015.02.022

26. Wang L, Damania B (2008) Kaposi's sarcoma-associated herpesvirus confers a survival advantage to endothelial cells. Cancer Res 68:4640-4648 . doi: 10.1158/0008-5472.CAN-07-5988

27. Appleton MA, Attanoos RL, Jasani B (1996) Thrombomodulin as a marker of vascular and lymphatic tumours. Histopathology 29:153-157

28. Kang H, Lieberman PM (2011) Mechanism of glycyrrhizic acid inhibition of Kaposi's sarcoma-associated herpesvirus: disruption of CTCF-cohesin-mediated RNA polymerase II pausing and sister chromatid cohesion. J Virol 85:11159-11169 . doi: 10.1128/JVI.00720-11

29. El Assal R, Gurkan UA, Chen P, et al (2016) 3-D Microwell Array System for Culturing Virus Infected Tumor Cells. Sci Rep 6:39144 . doi: 10.1038/srep39144

30. Herrmann J, Fayad AA, Muller R (2017) Natural products from myxobacteria: novel metabolites and bioactivities. Nat Prod Rep 34:135-160 . doi: 10.1039/c6np00106h

31. Mariggiò G, Koch S, Schulz TF (2017) Kaposi sarcoma herpesvirus pathogenesis. Philos Trans R Soc B Biol Sci 372:20160275 . doi: 10.1098/rstb.2016.0275

32. Haq I-U, Dalla Pria A, Papanastasopoulos P, et al (2016) The clinical application of plasma Kaposi sarcoma herpesvirus viral load as a tumour biomarker: results from 704 patients. HIV Med 17:56-61 . doi: 10.1111/hiv.12273

33. Mutlu AD, Cavallin LE, Vincent L, et al (2007) In vivo growth-restricted and reversible malignancy induced by Human Herpesvirus-8/ KSHV: a cell and animal model of virally induced Kaposi’s sarcoma. Cancer Cell $11: 245-258$

34. Cloutier N, van Eyll O, Janelle M-E, et al (2008) Increased tumorigenicity of cells carrying recombinant human herpesvirus 8. Arch Virol 153:93-103 . doi: 10.1007/s00705-007-1072-4 
35. Zhang J, Zhu L, Lu X, et al (2015) Recombinant Murine Gamma Herpesvirus 68 Carrying KSHV G Protein-Coupled Receptor Induces Angiogenic Lesions in Mice. PLoS Pathog 11: . doi: 10.1371/journal.ppat.1005001

36. An F, Folarin HM, Compitello N, et al (2006) Long-Term-Infected Telomerase-Immortalized Endothelial Cells: a Model for Kaposi's Sarcoma-Associated Herpesvirus Latency In Vitro and In Vivo. J Virol 80:4833-4846 . doi: 10.1128/JVI.80.10.4833

37. Blacher S, Erpicum C, Lenoir B, et al (2014) Cell Invasion in the Spheroid Sprouting Assay: A Spatial Organisation Analysis Adaptable to Cell Behaviour. PLoS One 9:e97019 . doi: 10.1371/journal.pone.0097019

38. Qin Z, Dai L, Toole B, et al (2011) Regulation of Nm23-H1 and cell invasiveness by Kaposi's sarcomaassociated herpesvirus. J Virol 85:3596-3606 . doi: 10.1128/JVI.01596-10

39. Dai L, Qiao J, Nguyen D, et al (2016) Role of heme oxygenase-1 in the pathogenesis and tumorigenicity of Kaposi's sarcoma-associated herpesvirus. Oncotarget 7:10459-10471 . doi: 10.18632/oncotarget.7227

40. Liu R, Gong M, Li X, et al (2010) Induction, regulation, and biologic function of Axl receptor tyrosine kinase in Kaposi sarcoma. Blood 116:297-305 . doi: 10.1182/blood-2009-12-257154

41. Jones T, Ramos da Silva S, Bedolla R, et al (2014) Viral cyclin promotes KSHV-induced cellular transformation and tumorigenesis by overriding contact inhibition. Cell Cycle 13:845-858 . doi: $10.4161 /$ cc. 27758

42. Aoki Y, Jaffe ES, Chang Y, et al (1999) Angiogenesis and hematopoiesis induced by Kaposi's sarcomaassociated herpesvirus-encoded interleukin-6. Blood 93:4034-4043

43. Dong X, Cheng A, Zou Z, et al (2016) Endolysosomal trafficking of viral G protein-coupled receptor functions in innate immunity and control of viral oncogenesis. Proc Natl Acad Sci 113:2994-2999 . doi: $10.1073 /$ pnas. 1601860113

44. Jensen KK, Manfra DJ, Grisotto MG, et al (2005) The Human Herpes Virus 8-Encoded Chemokine Receptor Is Required for Angioproliferation in a Murine Model of Kaposi’s Sarcoma. J Immunol 174:3686 LP-3694

45. Grisotto MG, Garin A, Martin AP, et al (2006) The human herpesvirus 8 chemokine receptor vGPCR triggers autonomous proliferation of endothelial cells. J Clin Invest 116:1264-1273 . doi: 10.1172/JCI26666 
46. Prakash O, Tang Z-Y, Peng X, et al (2002) Tumorigenesis and aberrant signaling in transgenic mice expressing the human herpesvirus-8 K1 gene. J Natl Cancer Inst 94:926-935

47. Bala K, Bosco R, Gramolelli S, et al (2012) Kaposi's Sarcoma Herpesvirus K15 Protein Contributes to Virus-Induced Angiogenesis by Recruiting PLC $\gamma 1$ and Activating NFAT1-dependent RCAN1 Expression. PLoS Pathog 8: . doi: 10.1371/journal.ppat.1002927

48. Gramolelli S, Weidner-Glunde M, Abere B, et al (2015) Inhibiting the Recruitment of PLC $\gamma 1$ to Kaposi’s Sarcoma Herpesvirus K15 Protein Reduces the Invasiveness and Angiogenesis of Infected Endothelial Cells. PLoS Pathog 11: . doi: 10.1371/journal.ppat.1005105

49. Braig S, Wiedmann RM, Liebl J, et al (2014) Pretubulysin: a new option for the treatment of metastatic cancer. Cell Death Dis 5:e1001 . doi: 10.1038/cddis.2013.510

50. O’Reilly T, McSheehy PMJ, Wenger F, et al (2005) Patupilone (epothilone B, EPO906) inhibits growth and metastasis of experimental prostate tumors in vivo. Prostate 65:231-240 . doi: 10.1002/pros.20289

51. Menhofer MH, Bartel D, Liebl J, et al (2014) In vitro and in vivo characterization of the actin polymerizing compound chondramide as an angiogenic inhibitor. Cardiovasc Res 104:303-314 . doi: 10.1093/cvr/cvu210 


\section{$611 \quad$ Figure legends}

612 Figure 1. KSHV establishes latency in conditionally immortalized human endothelial cells. a)

613 Immunofluorescence of rKSHV-HuARLT cells upon staining for viral LANA (red), green fluorescence is 614 a consequence of virus encoded GFP, DAPI staining was used to visualize the nucleus. b) FACS analysis 615 of non-infected control HuARLT cells and rKSHV-HuARLT cells before (D0) and after 16 days of 616 cultivation in absence of puromycin (D16). c) Viral copy number of rKSHV-HuARLT cells before (D0) 617 and after culture for 14 and 38 days in absence of puromycin. KF-HuARLT cells represent the GFP 618 negative cell population sorted from D38 rKSHV-HuARLT cells (see Fig. S1). The bars represent the 619 average value of 3 experiments with at least 3 independent replicates analyzed per experiment. The error 620 bars indicate standard deviation. Statistical significance was determined by t-test $* * p \leq 0.01$. d) mRNA levels of the indicated latent and lytic viral genes in rKSHV-infected HuARLT cells were determined via RT-qPCR and related to the mRNA levels of the cellular ACTB housekeeping gene. The experiment was performed 3 times with comparable outcomes. The graph shows mean and standard deviation of one representative experiment with 3 independent biological replicates. e) Cell viability upon cultivation of non-infected HuARLT and infected rKSHV-HuARLT cells with the indicated inhibitors for $72 \mathrm{~h}$ was accessed by WST cell viability assay. n.d.: non determined. The experiment was performed 3 times with comparable outcomes. The graph shows mean and standard deviation in one representative experiment with 3 biological replicates.

Figure 2. Characterisation of virus-infected (rKSHV-HuARLT), non-infected (HuARLT) and purged (KF-HuARLT) cells upon transplantation. a) Experimental setup. b) Lesions obtained from rKSHV-

632 HuARLT cells 4 weeks after transplantation were stained for hematoxyline and eosine, for ALU-positive nuclei as well as for GFP, LANA and CD141 expression. Representative immunohistochemistry sections 
are shown, magnification 250x. c) Representative pictures of engrafted cells isolated 4 weeks after transplantation upon staining for human vimentin. Scale bar $100 \mu \mathrm{m}$.

Figure 3. rKSHV-HuARLT based in vitro assays for compound validation. a) Mean fluorescence intensity (MFI) and relative viral copy number in rKSHV-HuARLT cells in 2D culture upon treatment with $25 \mu \mathrm{M}$ glycyrrhizic (GA) or $100 \mu \mathrm{M}$ phosphonoformic (PhA) acid for 14 days in the absence of puromycin. The experiment was performed 2 times with comparable outcomes. The graph shows mean and standard deviation in one representative experiment with 3 biological replicates. Statistical significance was determined by $\mathrm{t}$ test and is indicated by asterisks ${ }^{* *} \mathrm{p} \leq 0.01$. b) Relative viral copy number in rKSHV-HuARLT cells in 3D culture upon treatment with GA or PhA for 14 days. The experiment was performed 2 times with comparable outcomes. The graph shows mean and standard deviation in one representative experiment with 3 biological replicates. Statistical significance was determined by $\mathrm{t}$ test and is indicated by asterisks $* * \mathrm{p} \leq 0.01$. c) Relative sprouting index upon treatment of rKSHV-HuARLT cells with GA, PhA, Rapamycin or FK506 for 5 days. The graph shows mean and standard deviation of the pooled data from 3 independent experiments with at least 3 biological replicates analyzed in each condition. Statistical significance is indicated by asterisks: ${ }^{*} \mathrm{p} \leq 0.05,{ }^{* *} \mathrm{p} \leq 0.01,{ }^{* * *} \mathrm{p}$ $\leq 0.001,{ }^{* * * *} \mathrm{p} \leq 0.0001$. d) Phase contrast and fluorescent microscopy of representative 3D spheroids 5 days after embedding and treatment with GA, PhA, $2,5 \mu \mathrm{g} / \mathrm{ml}$ rapamycin, or $2,5 \mu \mathrm{g} / \mathrm{ml} \mathrm{FK506.}$

Figure 4. Evaluating novel compounds for viral loss and cellular invasiveness. a) Natural compounds inhibiting KSHV lytic reactivation in BJAB cells. A library of natural compounds was screened for the ability to reduce the production of infectious virus by lytically induced KSHV-infected BJAB (Brk.219) cells. The amount of infectious virus released from Brk.219 cells was measured by plating supernatants on HEK293 cells and measuring the GFP signal in a BioTek plate reader. Replicates are depicted. (b-e) Selected compounds were tested on rKSHV-HuARLT cells. The relative viral load was assessed in 
rKSHV-HuARLT cells upon culture in presence of the indicated drugs in standard 2D conditions (b) or in 3D matrigel (c). Non-treated cells were included as reference (control). $40 \%$ viral copy reduction in viral copy number was chosen as a cut-off value to select active compounds (dashed line). d) rKSHV-HuARLT cells were treated with the compounds in 3D cell culture conditions. The relative sprouting index (see Fig. S3) was determined after 2 weeks treatment. $40 \%$ reduction on sprouting index viral copy reduction was chosen as a cut-off value for active compounds (dashed line). For drug concentrations used in this experiment see Supplementary Table 1. Control compounds are indicated in green. The experiments in b, $\mathrm{c}$ and $\mathrm{d}$ were performed 2 times. The graphs show mean and standard deviation of representative experiments with 3-4 biological replicates per experiment. Statistical significance was determined by $t$ test and is indicated by asterisks: ${ }^{*} \mathrm{p} \leq 0.05,{ }^{* *} \mathrm{p} \leq 0.01,{ }^{* * *} \mathrm{p} \leq 0.001,{ }^{* * * *} \mathrm{p} \leq 0.0001$. e) Heat map summarizing the activity of the compounds in indicated assays.

Figure 5. Compound validation in vivo. a) Representative immunohistochemistry sections from lesions observed without treatment or upon 4 weeks treatment with indicated compounds. Cells were stained for human vimentin, scale bar $200 \mu \mathrm{m}$. b) The lesion size was measured and is indicated as $\%$ of non-treated control ( $\mathrm{n}=6$ per compound). $25 \%$ tumor size reduction was chosen as a cut-off value for active compounds. Statistical significance is shown by asterisks: $* \mathrm{p} \leq 0.05,{ }^{* *} \mathrm{p} \leq 0.01, * * * \mathrm{p} \leq 0.001, * * * * \mathrm{p}$ $\leq 0.0001$, (10 to 80 lesions in 6 plugs). c) Correlation analysis of 3D sprouting index vs. lesion size diameter in vivo. Scale bar $200 \mu \mathrm{m}$. 

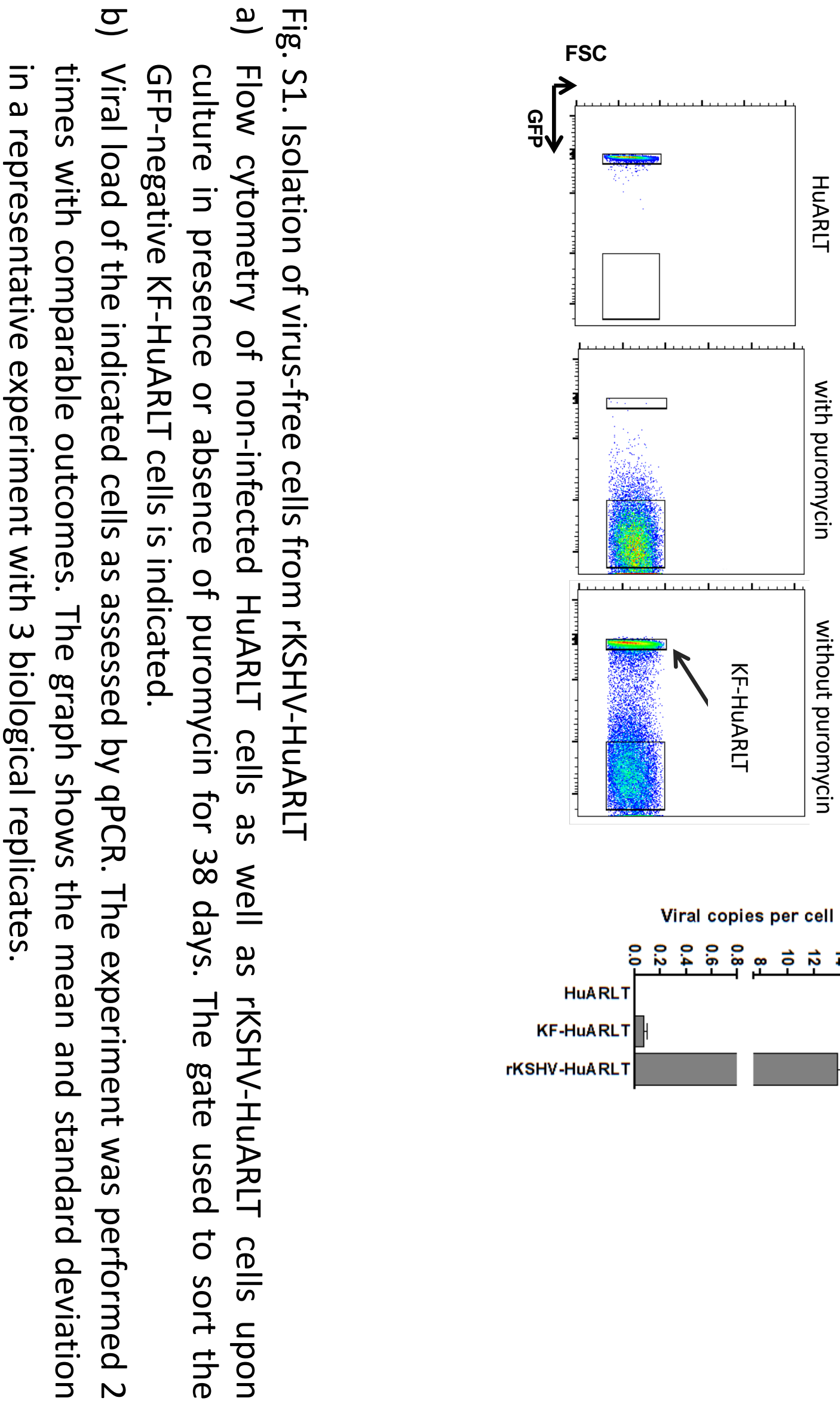

ㄴ

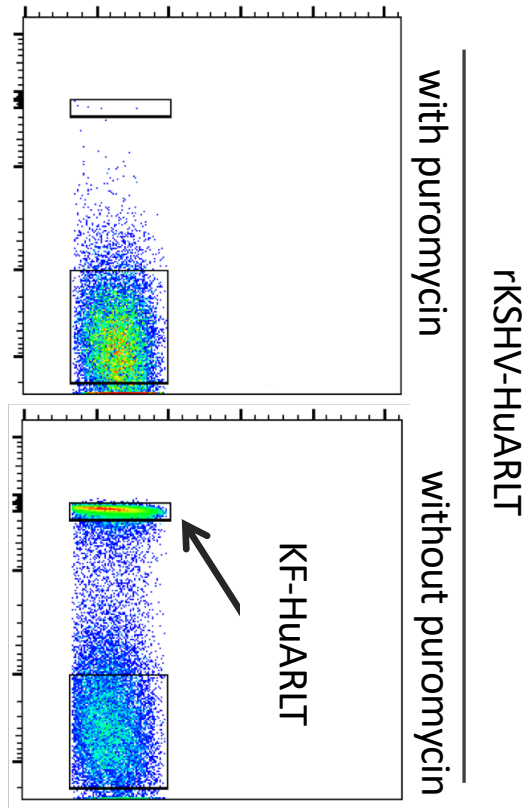

$\underline{0}$

Viral copies per cell

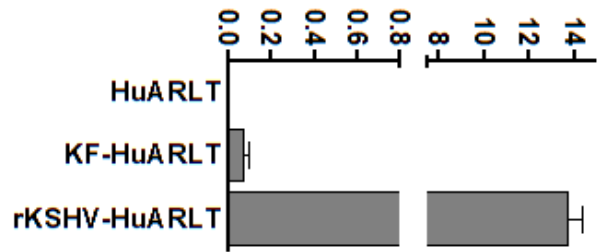

5

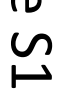




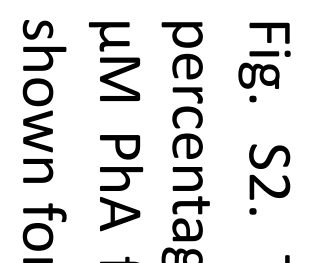

吕

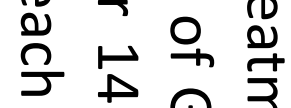

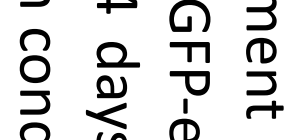

을 会

$\nabla \stackrel{n}{J} \Omega$

กิ $\stackrel{0}{\supset} D$

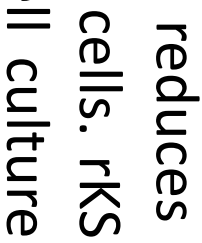

Oे $\stackrel{1}{<} \stackrel{0}{<}$

ᄁก rKSHV-HuARLT

$\frac{1}{10}$

N

오 임

$\sum_{D} \frac{5}{D}$

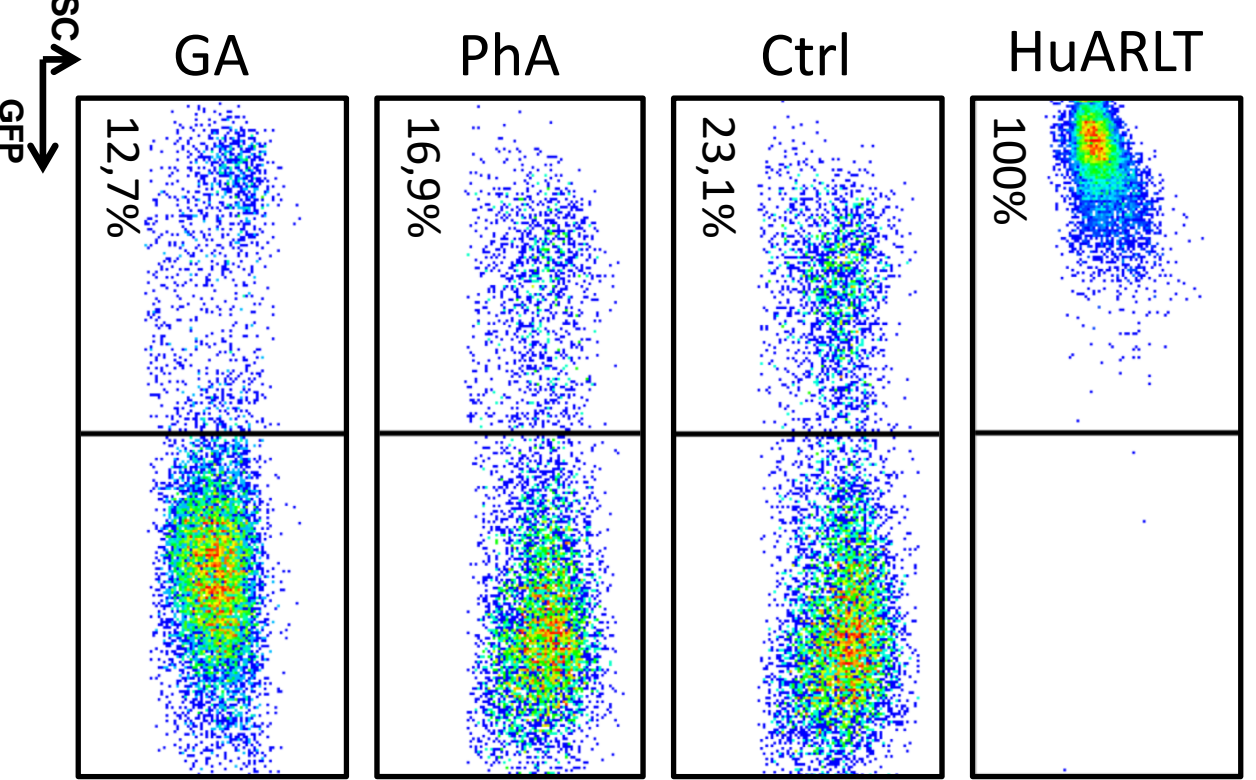

르

만 D

고 $\frac{D}{\bar{n}} \frac{0}{D}$

คิ $\sum_{0} \frac{n}{2}$.

Ð $\frac{D}{D}$

슷 웅

ज. 俩 苗

D

票市

命 N

ग $\mathrm{D} \cong$

总 ह

응하 굼

爱客宽

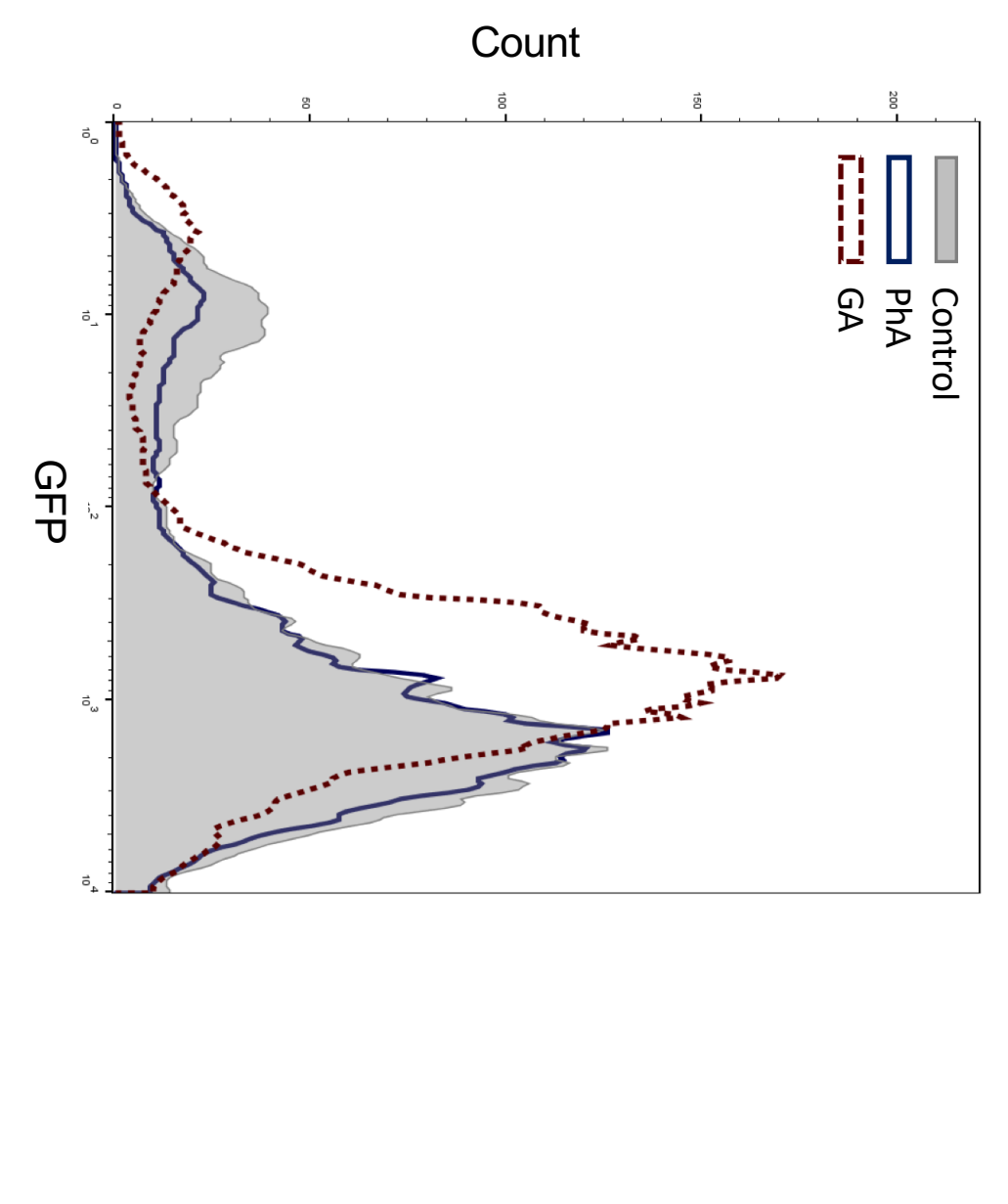



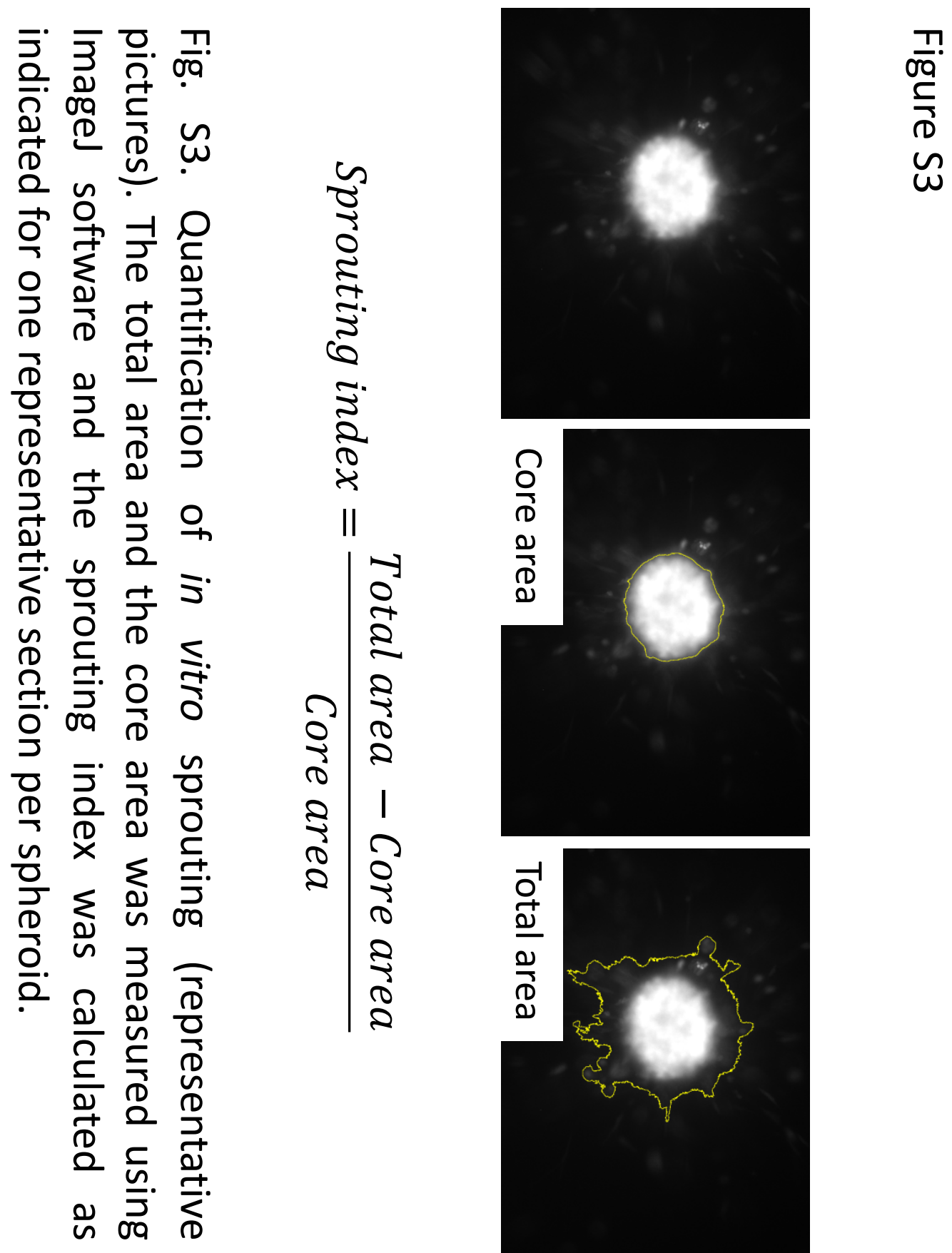

$\frac{7}{09}$
$\frac{c}{0}$
$\stackrel{D}{\omega}$ 


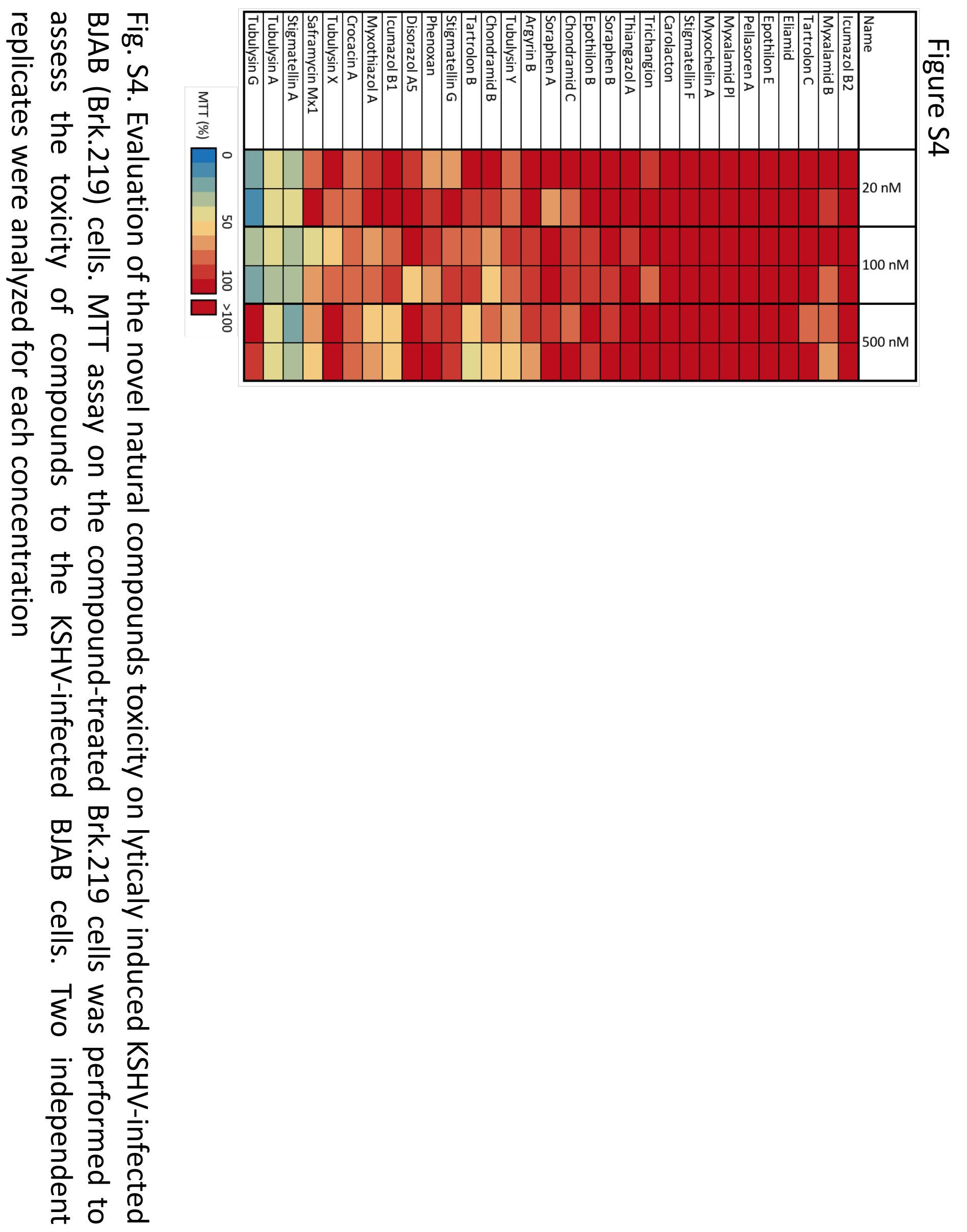




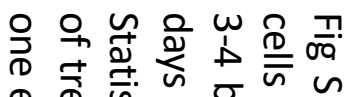

Q

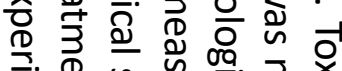

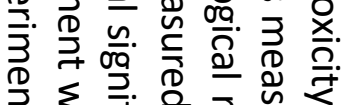

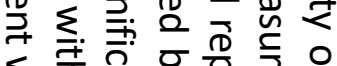

ㄴ. 웡

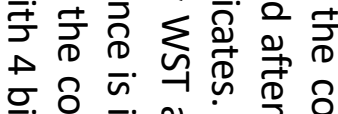

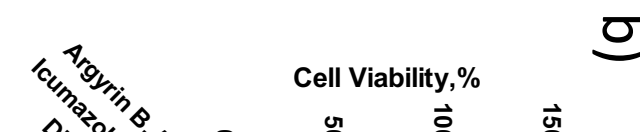

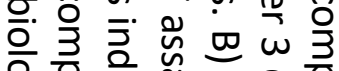

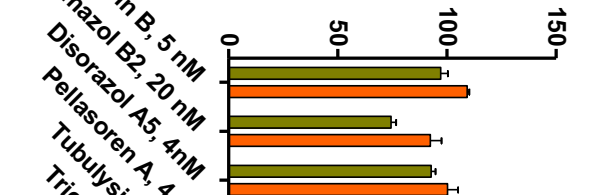

$\sigma$

v

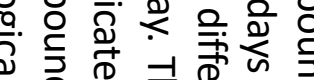

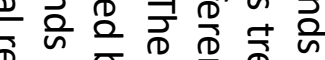

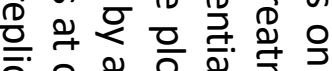

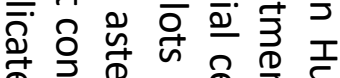

类

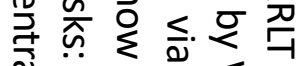

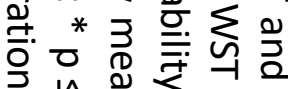

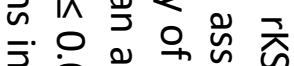

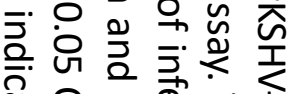

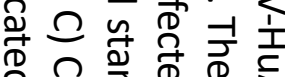

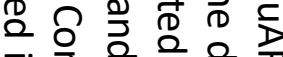

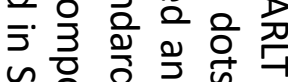

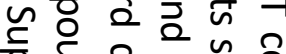

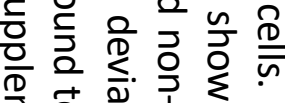

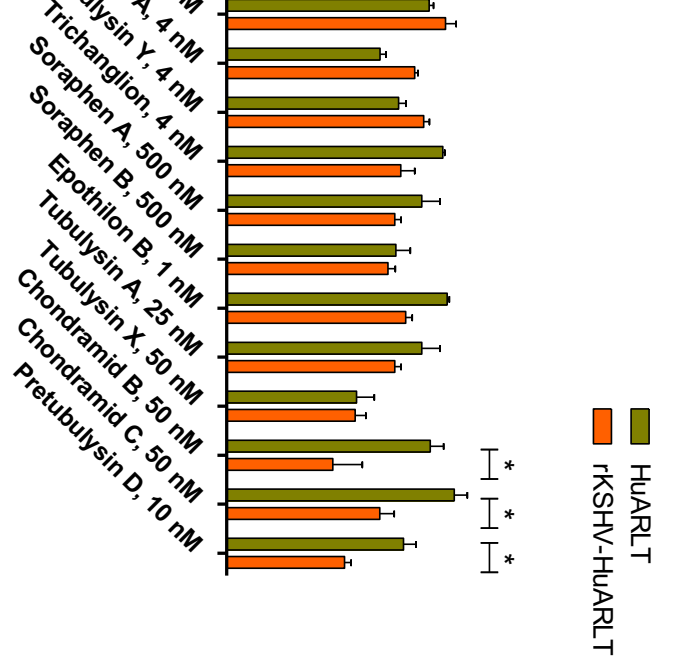

Cell Viability,\%
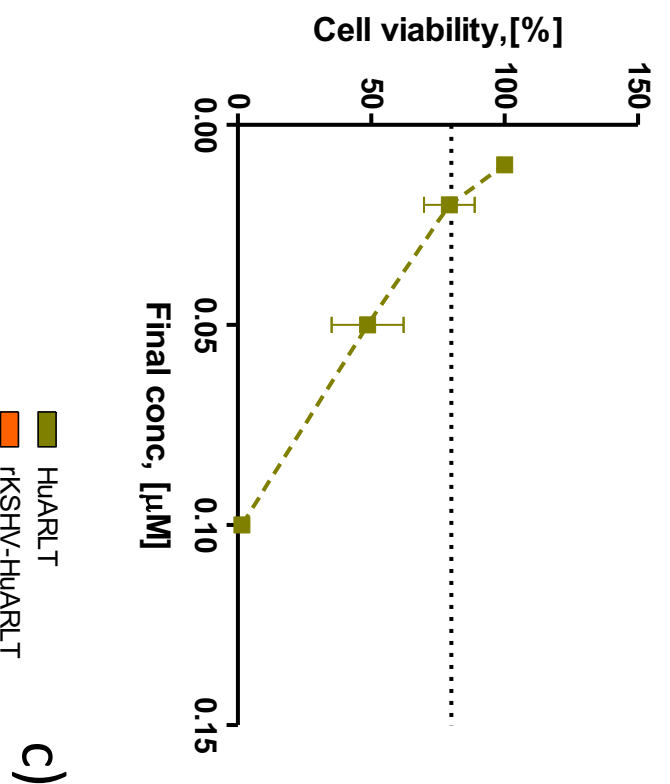

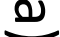

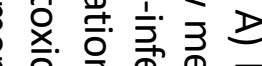

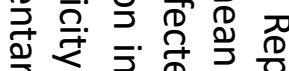

$<0$ 잉

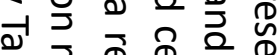

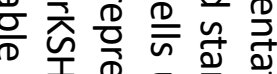

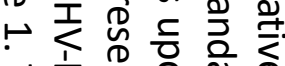

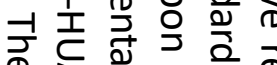

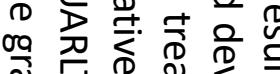

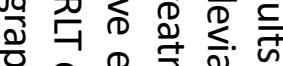

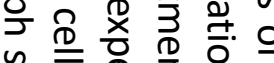

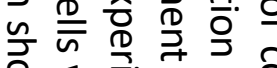

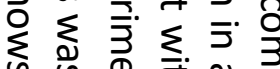

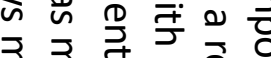

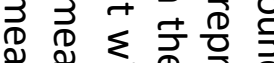

ขึ

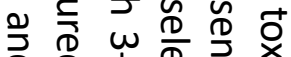

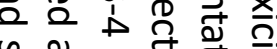

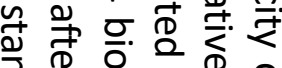

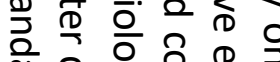

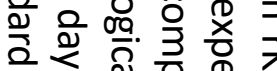

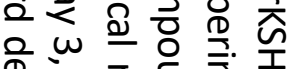

证更产

ㄴ.

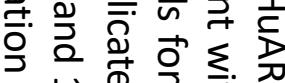

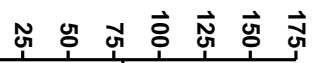
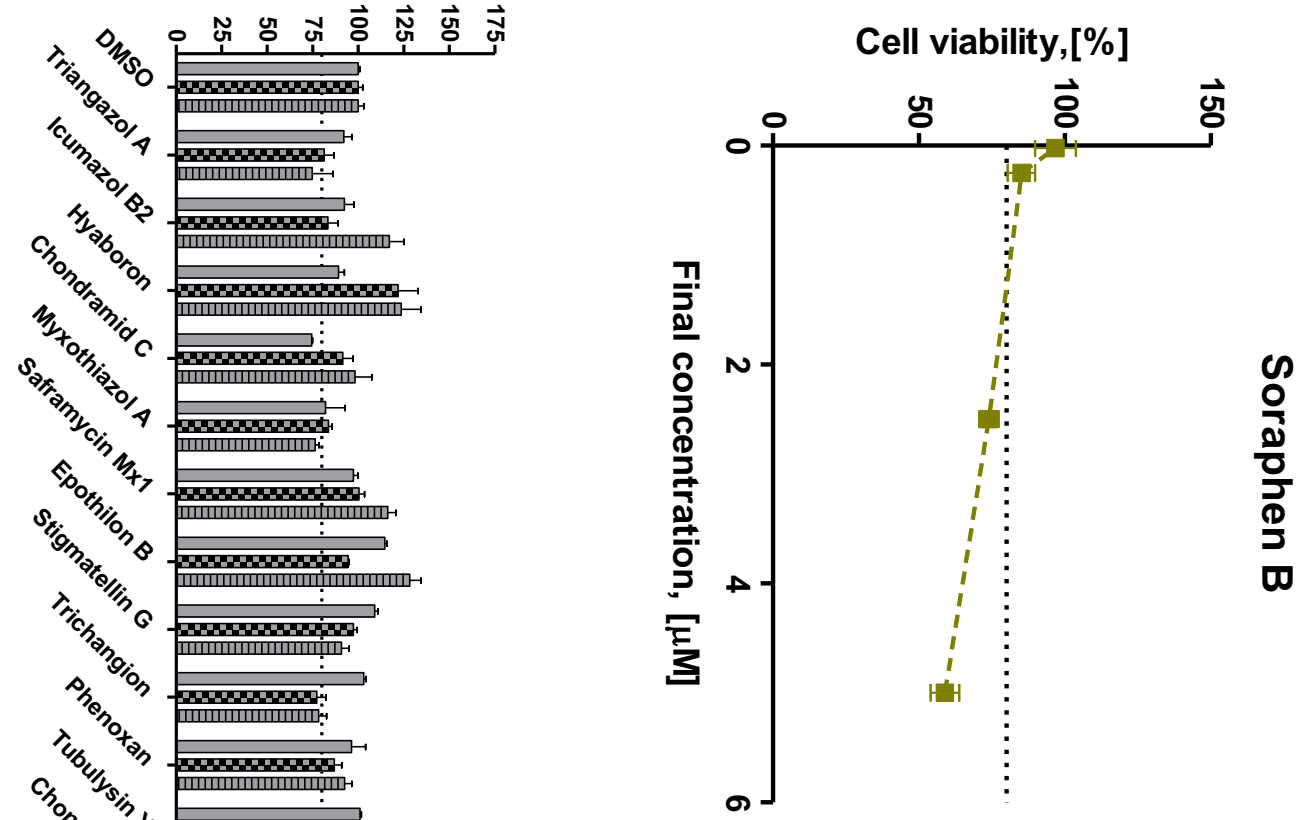

웅

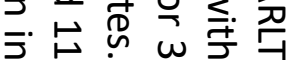




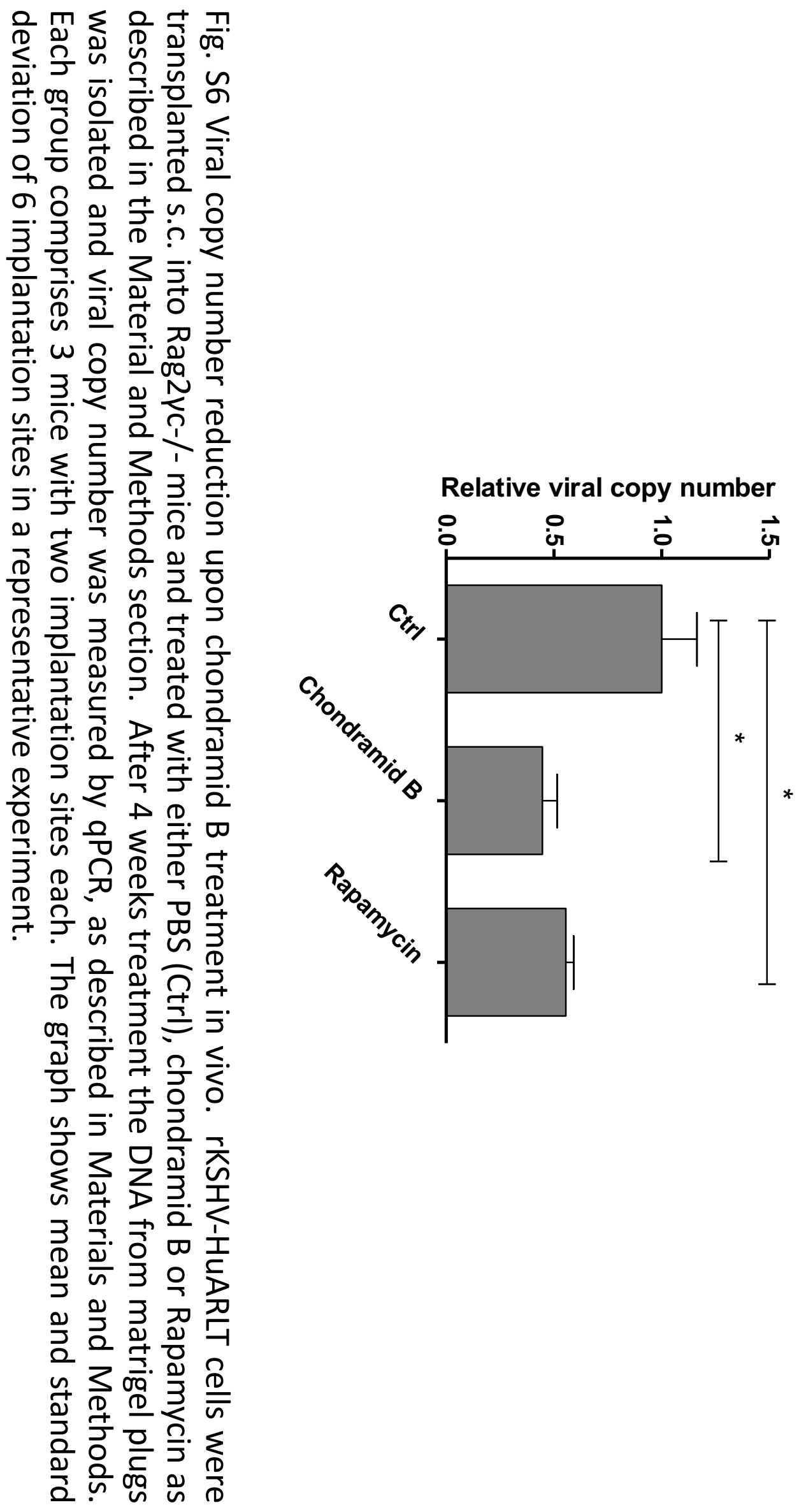




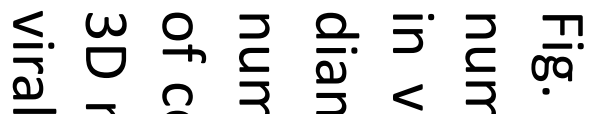

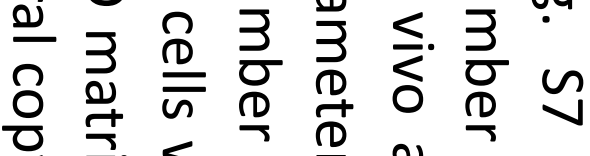

穴市

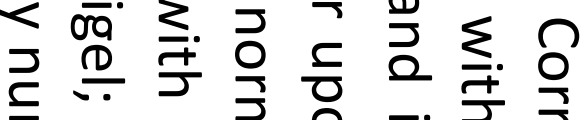

完气 。

은 윽 吾

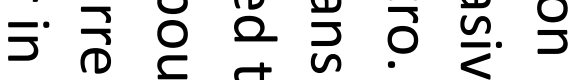

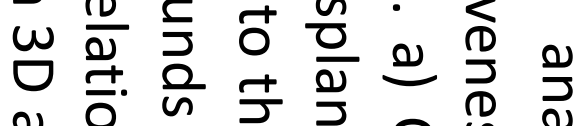

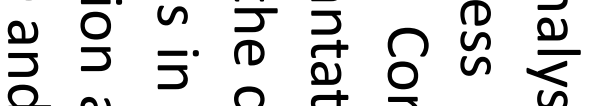

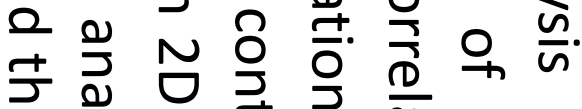

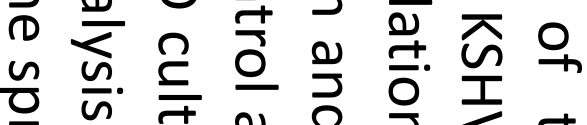

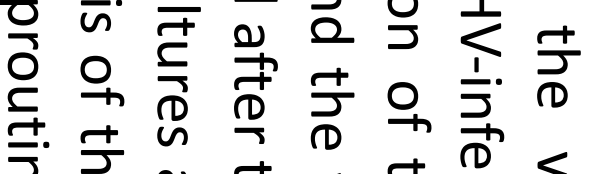

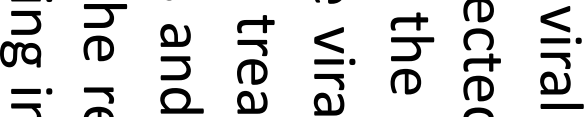

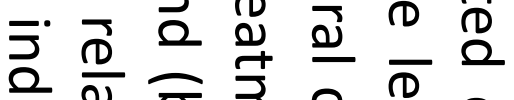

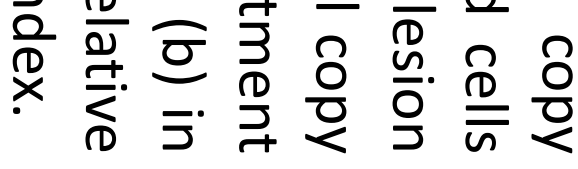
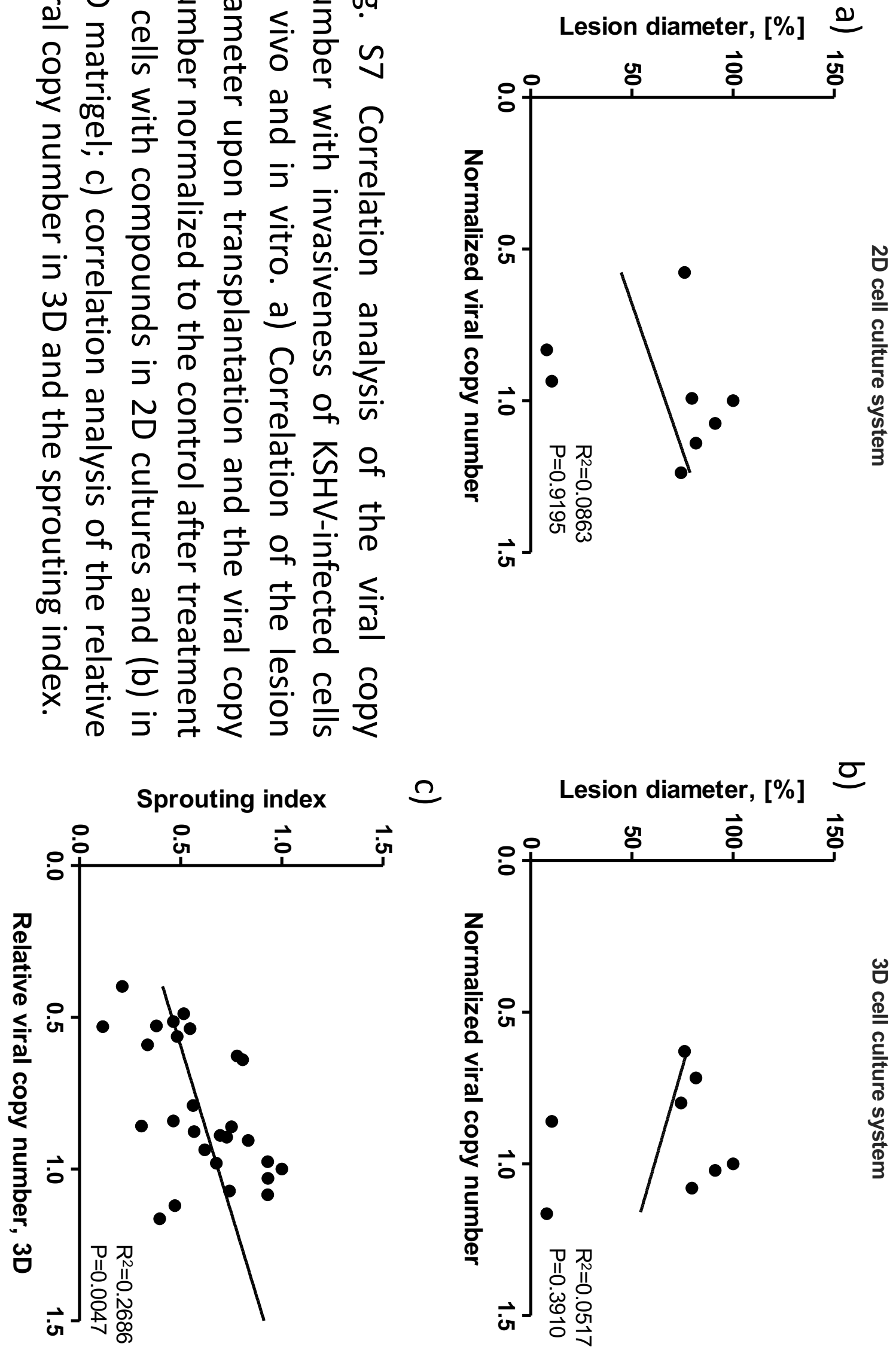


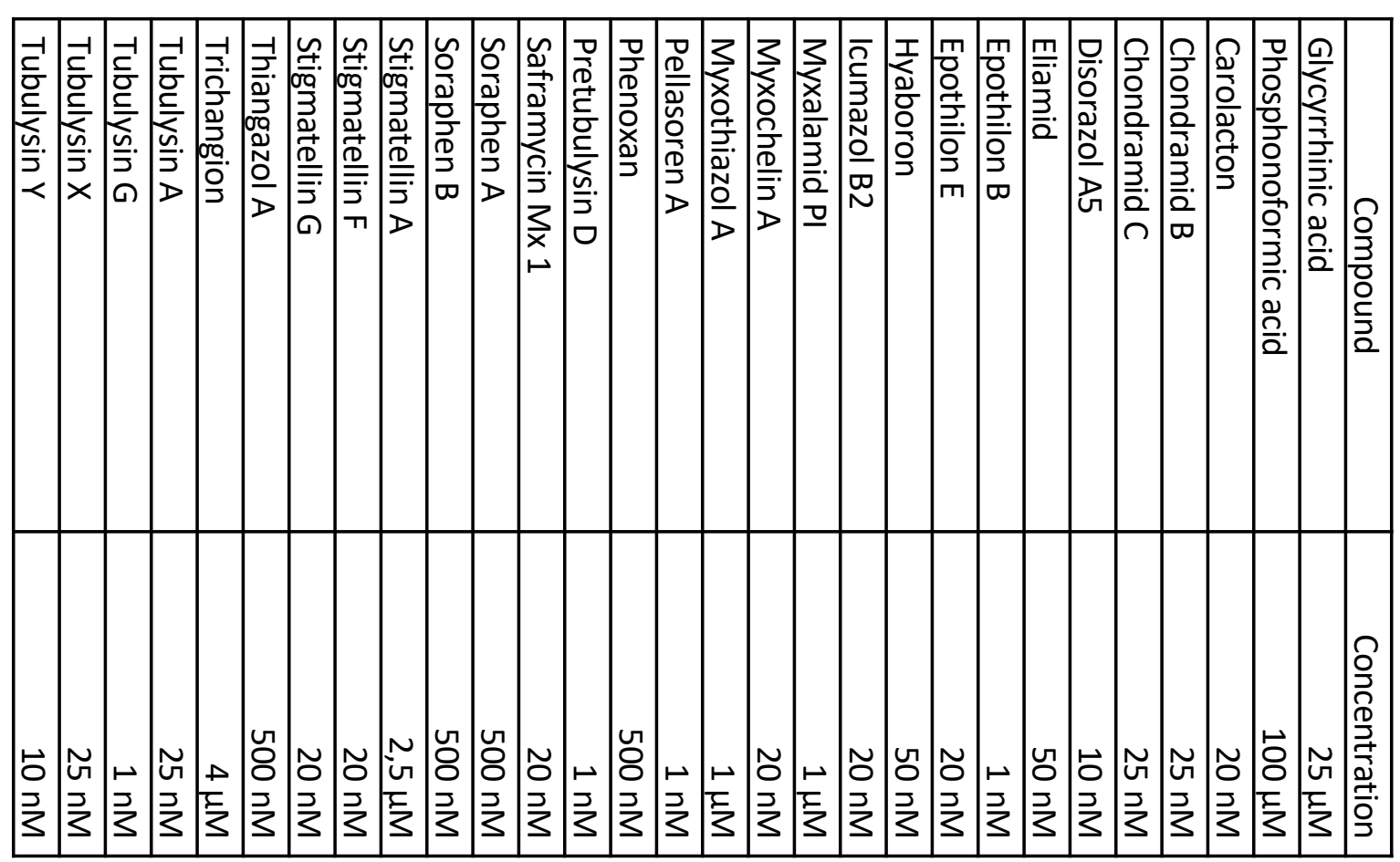

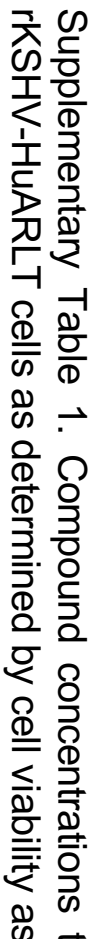

兽宾

¿ำ

금

腺

융

을. 


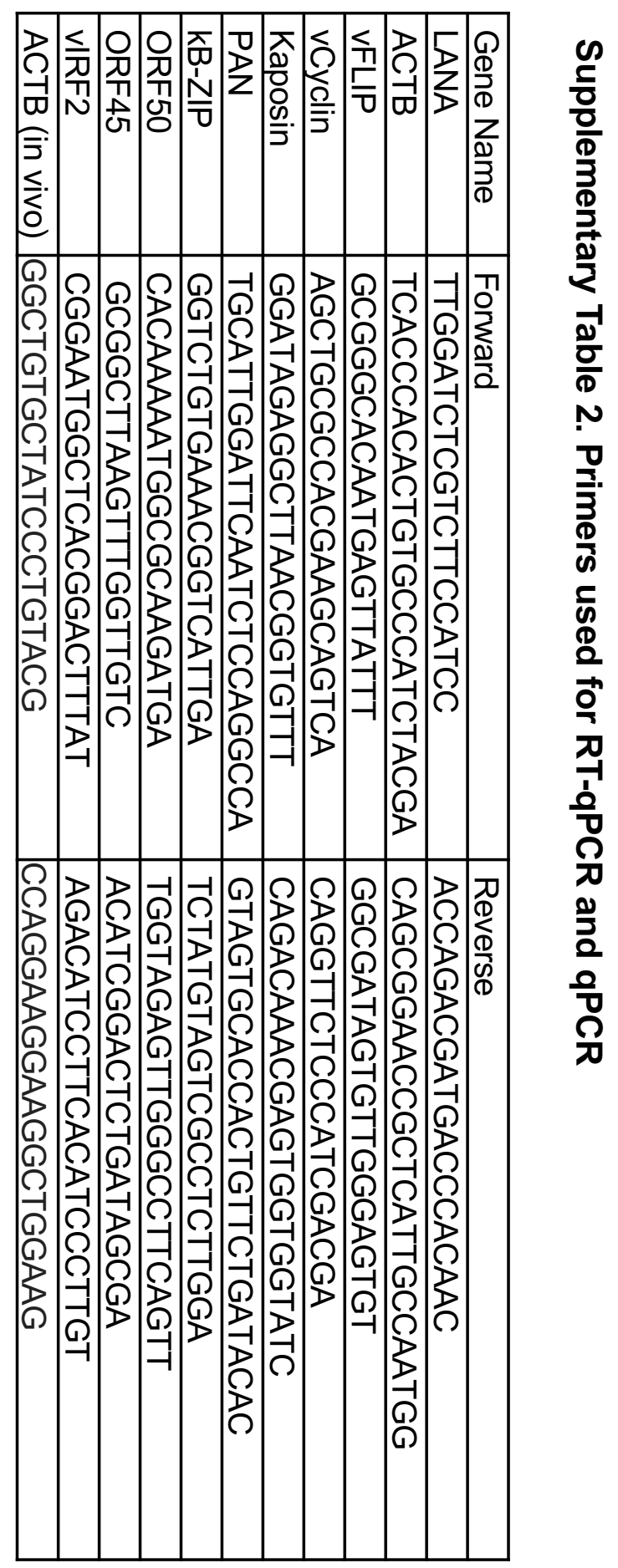




\begin{tabular}{|c|c|c|c|c|c|c|c|}
\hline 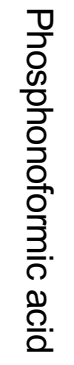 & 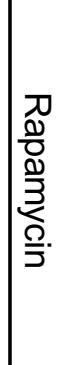 & 商 & 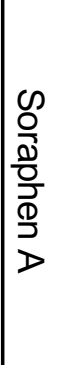 & 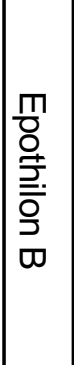 & 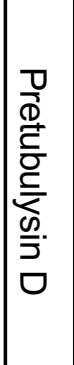 & 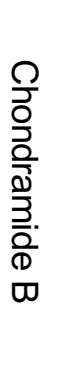 & $\begin{array}{l}\text { ᄋ్ } \\
\frac{3}{0} \\
\frac{0}{0} \\
\frac{\bar{z}}{2}\end{array}$ \\
\hline $\bar{\circ}$ & $\bar{\circ}$ & $\overline{0}$ & 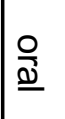 & 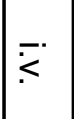 & $\ddot{<}$ & $\dot{<}$ & $\begin{array}{l}\text { DD } \\
\stackrel{0}{\bar{巾}}\end{array}$ \\
\hline$\widetilde{8}$ & $\rightarrow$ & $N$ & 잉 & $\hat{\tilde{\sigma}}$ & $\rightarrow$ & or & 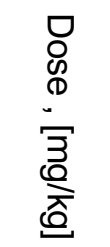 \\
\hline 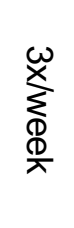 & 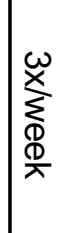 & 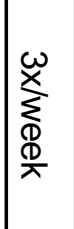 & 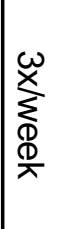 & 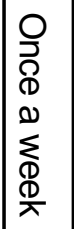 & 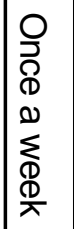 & 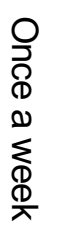 & 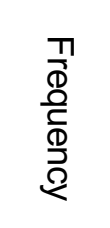 \\
\hline $\begin{array}{l}\text { 怘 } \\
\text { C. }\end{array}$ & 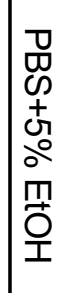 & 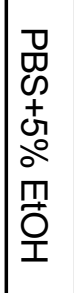 & 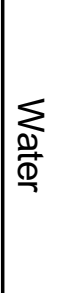 & 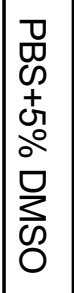 & 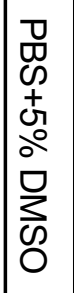 & 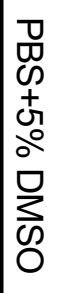 & $\begin{array}{l}\infty \\
\stackrel{\infty}{\Lambda} \\
\stackrel{\Phi}{=}\end{array}$ \\
\hline $\overrightarrow{8}$ & $\overrightarrow{8}$ & $\overrightarrow{8}$ & $\overrightarrow{8}$ & $\overrightarrow{8}$ & $\overrightarrow{8}$ & $\overrightarrow{8}$ & 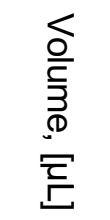 \\
\hline$\Phi$ & $\Xi$ & $\Xi$ & $\sigma$ & $\begin{array}{l}F \\
\text { or }\end{array}$ & $\mid \begin{array}{c}\tilde{N} \\
\omega \\
\omega\end{array}$ & $\Xi$ & 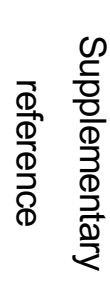 \\
\hline
\end{tabular}

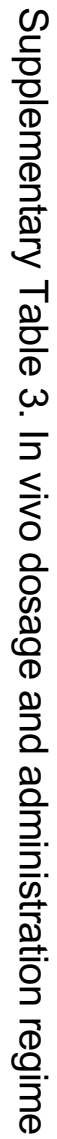




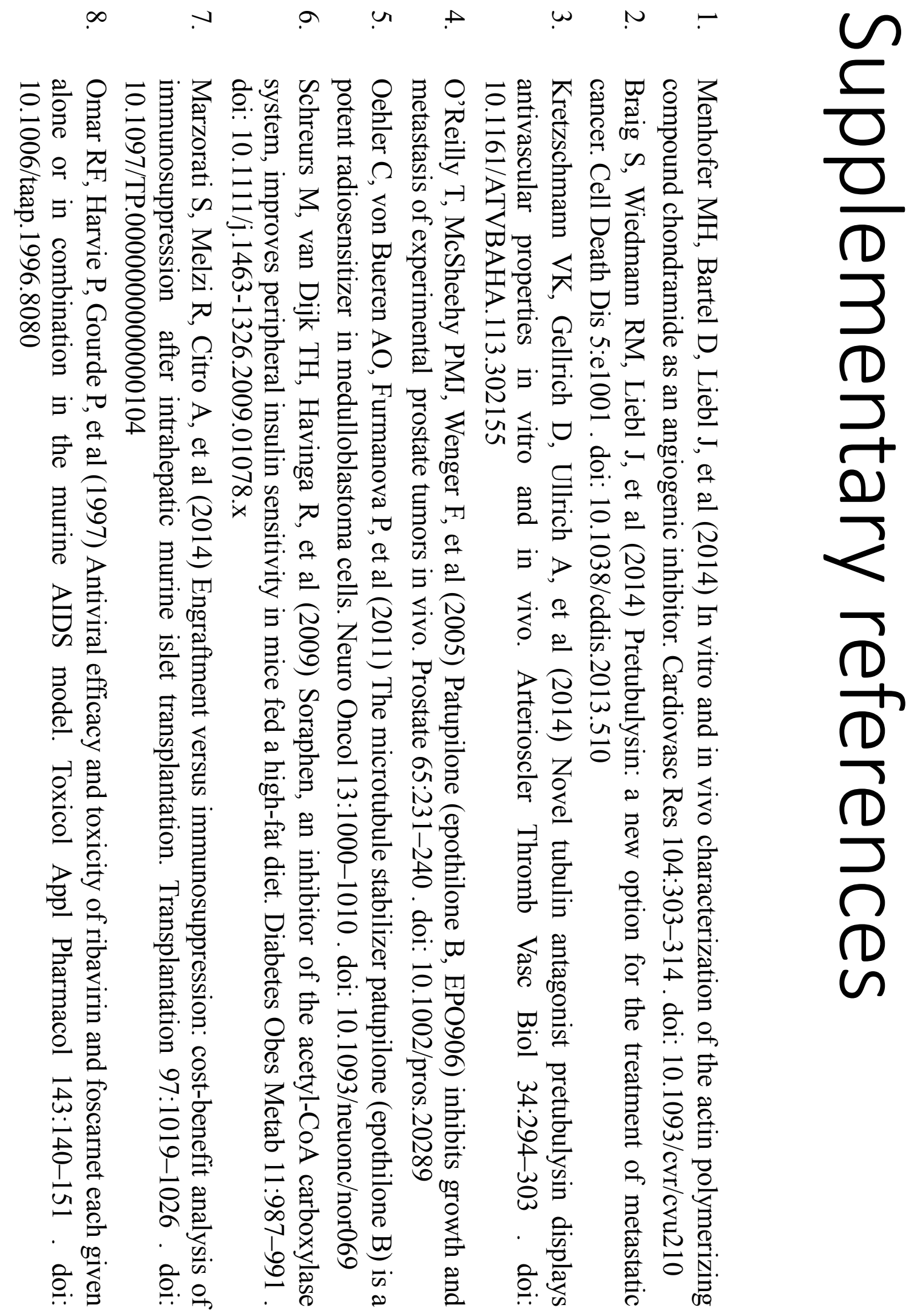

